\title{
Algebraic Representation of Dynamics and Behavior for Continuous-Time Linear Systems
}

\author{
Stephen J. Hegner \\ Department of Computer Science and Electrical Engineering \\ Votey Building \\ University of Vermont \\ Burlington, VT 05405 \\ (802)656-3330 \\ hegner@uvm.edu \\ ..!uunet!uvm-gen!hegner
}

This paper appeared in Mathematical Systems Theory, 22(1989), pp. 227-254.

Keywords: algebraic system theory, dynamics, behavior, semigroups of operators, continuous-time systems

\begin{abstract}
An algebraic approach to continuous-time linear systems is presented which closely parallels the discrete-time decomposable systems approach of Arbib and Manes, as well as the older $k[z]$ module theory of linear systems of Kalman. The focal point of the presentation is a class of topological rings, termed $\mathbf{R}_{+}$-rings, which play the same role for continuous time that $k[z]$ does for discrete-time. Each such ring $R$ defines a class of toplogical modules, termed the $(R)$-modules, which may be naturally identified with a class of locally equicontinuous semigroups, called the $(R)$ semigroups. Thus, just as discrete-time linear dynamics are coextensive with $k[z]$-modules, so too are continuous-time linear dynamics coextensive with $(R)$-modules. This identification underlies the development of a purely algebraic theory of behavior and realization for continuous-time linear systems. The specific choice of $R$ determines the type of dynamics allowed. For example, taking $R$ to be the ring of all measures on the nonnegative reals yields dynamics described by the class of all semigroups, while choosing $R$ to be the ring of all $L^{1}$ measures yields dynamics whose responses vanish at infinity.

A central focus of this paper is the construction of free and cofree dynamics, and hence the behavior, of a system. Just as a behavior in the discrete-time case may be thought of as a $k[z]$ module homomorphism from the free $k[z]$-module $k[z] \otimes I$ to the cofree $k[z]$-module $L(k[z], Y)$, so too may a behavior in the continuous-time case be viewed as suitably continuous $(R)$-module homomorphism from a suitable topologization and completion of $R \otimes I$ to a suitable topologization of the space $\mathbf{L}(R, Y)$ of continuous linear maps.
\end{abstract}




\section{Motivation - A Review of the Discrete-Time Concepts}

In providing a motivating overview of our presentation, let us begin by recalling some of the basic algebraic aspects of sequential machine behavior and realization. Let $M=(Q, \delta, I, Y, h)$ be a sequential machine. Here $Q$ is the state set, $I$ the input set, and $\delta: Q \times I \rightarrow Q$ the dynamics. $Y$ is the output set and $h: Q \rightarrow Y$ the output map. We may think of $M$ as being described by dynamical equations of the form

$$
\begin{aligned}
q(t+1) & =\delta(q(t), i(t)) \\
y(t) & =h(q(t)) .
\end{aligned}
$$

It is well known that a more global picture of the behavior may be obtained by extending $\delta$ to a right action $\delta^{*}: Q \times I^{*} \rightarrow Q$ of the free monoid $I^{*} . \beta \circ \delta: Q \times I^{*} \rightarrow Y$ then gives the behavior of the machine.

Placing into a categorical framework this process of extracting the behavior from the local dynamics has been the subject of a number of investigations, including that of Arbib and Manes ([AM1], [AM2], [AM3], [AM4], [AM5]), Bainbridge ([B1]), Ehrig and his co-workers ([EKKK], $[\mathrm{EK}]$ ) and Goguen ([G]). The following quick review (specialized to the above example of sequential machines) follows most closely the approach of Arbib and Manes. Fixing the set $I$, define the category $\operatorname{Dyn}(-\times I)$ of $-\times I$-dynamics to have as objects pairs of the form $(Q, \delta)$ with $\delta: Q \times I \rightarrow Q$; a morphism $k:(Q, \delta) \rightarrow\left(Q^{\prime}, \delta^{\prime}\right)$ (called a dynamorphism) is a function $k: Q \rightarrow Q^{\prime}$ such that $k \circ \delta=\delta^{\prime} \circ(k \times \mathbf{1})$. Consider the free $-\times I$-dynamics $\left(Q \times I^{*}, \mu\right)$ over $Q$, as defined by the following diagram.

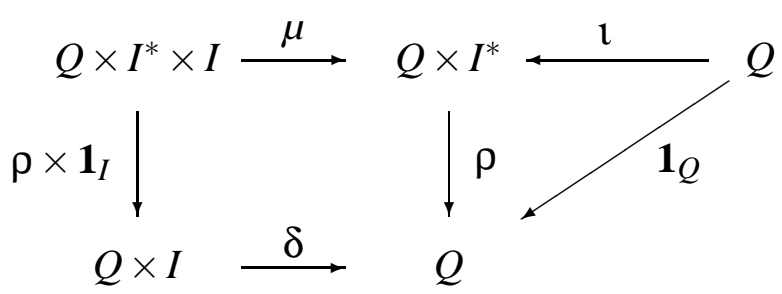

The free action $\mu: Q \times I^{*} \times I \rightarrow Q \times I^{*}$ is just concatenation $(q, w, a) \mapsto(q, w a) . \imath: q \mapsto(q, \lambda)$, where $\lambda$ is the empty string. $\rho$ is the unique reachability map, and is just the extension $\delta^{*}$ of $\delta$ to strings that we seek.

The dual construction for the output side is depicted in the diagram below. 


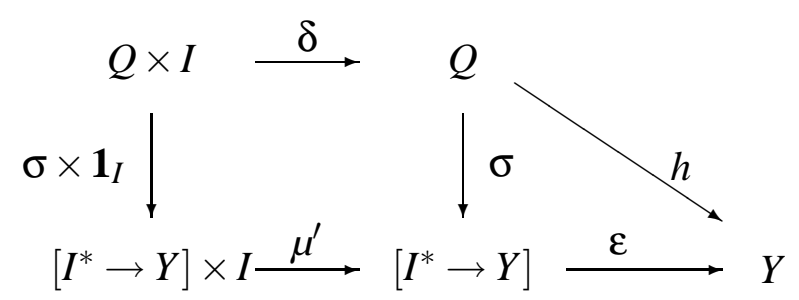

$\left[I^{*} \rightarrow Y\right]$ is the set of all functions from $I^{*}$ to $Y$. $\left(\left[I^{*} \rightarrow Y\right] \times I, \mu^{\prime}\right)$ is the cofree- $\times I$-dynamics over $Y ; \mu^{\prime}:(f, i) \mapsto f(i \cdot-) ; \varepsilon: f \mapsto f(\lambda)$. $\sigma$ is the unique observability map for $M ; \sigma(q)=\rho(q,-)$. $\sigma \circ \rho$ is the total behavior of $M$.

The above example illustrates a general categorical construction specialized to the category of sets and functions. Similar constructions work, with varying degrees of success, in several other categories. For details, consult [AM2]. The context which is of direct interest to us here is that of linear systems. In this case, we fix a (commutative, with unit) ring $\mathbf{K}$, and work within the category of K-modules and their homomorphisms (K-linear maps). Thus, relative to the set-based framework just described, sets become $\mathbf{K}$-modules, functions become $\mathbf{K}$-module homomorphisms, and the cartesian product $-\times-$ translates to the tensor product $-\otimes-$.

Unfortunately, this direct translation does not provide quite what we want. For consider a dynamics $\delta: Q \otimes I \rightarrow Q$. Linearity of this mapping means that $\delta(q, 0)=\delta(q \otimes 0)=0$ for any $q \in Q$. In other words, in such a dynamics, the zero input always drives the machine to the zero state in one step, which is quite unreasonable. Rather, the transition function of a linear system is typically taken to be of the form $\delta(q, i)=f(q)+g(i)$ for some linear $f: Q \rightarrow Q$ and $g: I \rightarrow Q$. Such a function is affine, but not linear. While we could develop a theory of linear systems based upon such affine transformations, there is an alternate approach which retains almost all of the characteristics of the set-based approach. Specifically, we take advantage of the decomposability of the state-transition map to yield the theory of decomposable system in a category of Arbib and Manes [AM1]. The following summarizes that approach for linear systems over the ring $\mathbf{K}$. (For another discussion of the relationship between the decomposable systems approach and the approach described above, see [AM4, sec. 6]). A (decomposable) linear system (in the category of K-modules) is a 6-tuple $M=(Q, f, I, g, Y, h)$ where $Q$ (the state space), $I$ (the input space), and $Y$ (the output space) are all K-modules, and $f: Q \rightarrow Q$ (the state-transition map), $g: I \rightarrow Q$ (the input map), and $h: Q \rightarrow Y$ (the output map) are all $\mathbf{K}$-linear. The dynamics are described by the equations

$$
\begin{aligned}
q(t+1) & =f(q(t))+g(i(t)) \\
y(t) & =h(q(t)) .
\end{aligned}
$$

Dynamics in the decomposable systems framework are with respect to the identity; thus, a dynamics is a pair $(Q, \delta)$ with $\delta: Q \rightarrow Q$, and a dynamorphism $k:(Q, \delta) \rightarrow\left(Q^{\prime}, \delta^{\prime}\right)$ is a $\mathbf{K}$-linear 
map $k: Q \rightarrow Q^{\prime}$ such that $k \circ \delta=\delta^{\prime} \circ k$. Just as a dynamics $(Q, \delta: Q \times I \rightarrow Q)$ in the set-based case gave rise to an action $\delta^{*}: Q \times I^{*} \rightarrow Q$, so too does a dynamics $(Q, \delta: Q \rightarrow Q)$ in the linear case provide such an action. The ring (qua monoid) in this case is $\mathbf{K}[z]$, the ring of polynomials over $\mathbf{K}$ in the single variable $z$. Multiplication is convolution. $\delta: Q \rightarrow Q$ defines the action on the simple polynomial $z$, with linearity and multiplication providing the others. The analogs of diagrams (2) and (3) are provided by the following.

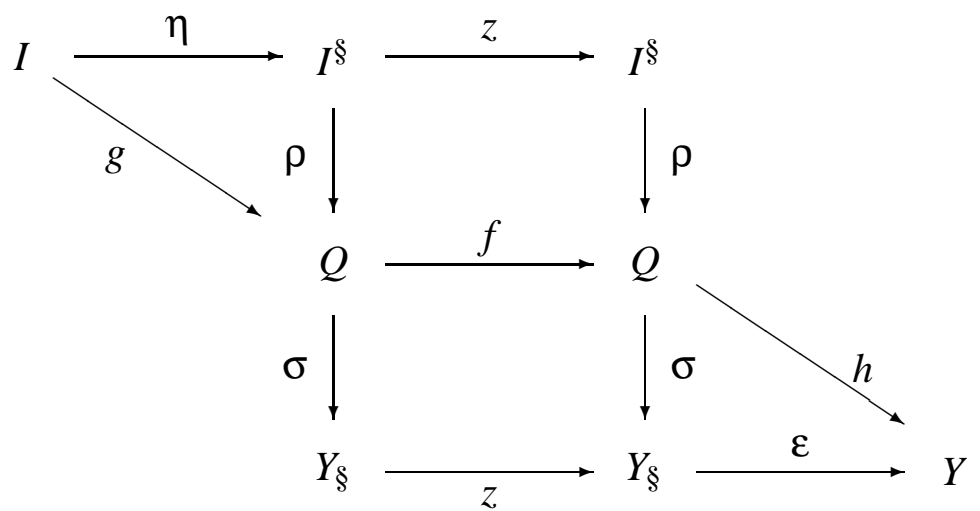

$\left(I^{\S}, z\right)$ is the free dynamics over $I . I^{\S}=\left\{\left(i_{0}, i_{1}, . ., i_{k}, \ldots\right) \mid i_{k}=0\right.$ for all but finitely many $\left.k\right\}$. $z:\left(i_{0}, i_{1}, \ldots\right) \mapsto\left(0, i_{0}, i_{1}, \ldots\right)$ is the right shift. $\eta: i \mapsto(i, 0,0, . ., 0, \ldots)$. It is not difficult to see that $I^{\S} \cong I \otimes \mathbf{K}[z]$. By specializing $I$ to be $Q$ and $g: Q \rightarrow Q$ to be the identity, we can explicitly recover the module action of $f$ as $\rho: Q^{\S} \cong Q \otimes \mathbf{K}[z] \rightarrow Q$. More generally, $\rho: I^{\S} \rightarrow Q$ is the reachability map of $M$, and tells us the state of $M$ at time 0 due to input $\left(i_{0}, i_{1}, \ldots\right) \in I^{\S}$, with $i_{k}$ occurring at time $-k$.

Similar ideas hold for the output side. $\left(Y_{\S}, z\right)$ is the cofree dynamics over $I . \quad Y_{\S}=$ $\left\{\left(y_{0}, y_{1}, . ., y_{k}, \ldots\right) \mid y_{k} \in Y\right\}$, with $z:\left(y_{0}, y_{1}, y_{2}, \ldots\right) \mapsto\left(y_{1}, y_{2}, \ldots\right)$ the left shift. $\varepsilon:\left(y_{0}, y_{1}, \ldots\right) \mapsto y_{0}$. Furthermore, $Y_{\S} \cong L(\mathbf{K}[z], Y)$ ( $L=$ linear maps). $\sigma$ is called the observability map, and $\sigma(q)$ is the output sequence observed when starting in state $\mathrm{q}$ with all further inputs 0 .

For $I=\mathbf{K}^{m}$ and $Y=\mathbf{K}^{p}$, the above framework is essentially Kalman's $\mathbf{K}[z]$-module approach $[\mathrm{K} 1]$.

\section{Continuous Time - The Basic Ideas}

Before presenting the mathematical details, we provide an informal overview of how these algebraic representations of the discrete-time case are extended to continuous time; in particular we highlight the critical differences which arise.

\section{Dynamics and Systems}

The major goal of this work is to extend the above constructions to the domain of continuous-time linear systems. In other words, the dynamics of equations (4) are to be replaced by 


$$
\begin{aligned}
d q(t) / d t & =f(q(t))+g(i(t)) \\
y(t) & =h(q(t)) .
\end{aligned}
$$

This immediately imposes some natural restrictions. First of all, $\mathbf{K}$ is now either $\mathbf{R}$ (the field of real numbers) or else $\mathbf{C}$ (the field of complex numbers), and $\mathbf{K}$-modules are locally convex topological vector spaces over $\mathbf{K}$. Thus, our underlying category $\mathcal{K}$ must be one whose objects are locally convex spaces, and whose morphisms are continuous linear mappings. In fact, everything in sight must be given a topological as well as algebraic flavor, and all constructions must be translated to this framework. In particular, any tensor products must be topological and module actions must posess some sort of continuity.

Assuming that we have established a working domain with these additional features, let us reexamine the nature of a dynamics under the interpretation (6). To admit unique solutions in the context of (6), we take $f$ to be the infinitesimal generator of a one-parameter semigroup of operators (the parameter being $\mathbf{R}_{+}$, the nonnegative reals). In an earlier paper [H2], we presented a categorical framework for continuous-time linear systems which dealt exclusively with infinitely differentiable systems; i.e., systems for which $f$ in (6) above is everywhere defined and continuous. Unfortunately, many examples occurring in practice do not have differentiable dynamics, and this shortcoming motivated, at least in part, the present paper. Here we take $f$ to be the infinitesimal generator of a locally equicontinuous semigroup of operators in the sense of Kōmura [K5]. On the other hand, to get a satisfactory algebraic characterization, we still require $g$ and $h$ to be continuous linear operators. However, this does not present an unrealistic constraint, since we allow distributions as inputs, and interpret the first equation of (6) in the following well-known operational sense, which effectively defines the reachability map $\sigma$.

$$
q(t)=e^{f t} \cdot x(0)+\int_{0}^{t} e^{f(t-s)} g(i(s)) d s
$$

In the above, $e^{f t}$ is semigroup generated by $f$, and the integral is interpreted as distributional convolution. The continuous-time analog $I^{\$}$ of $I^{\S}$ (from which the input signal $i(s)$ is taken) is first approximated as finite linear combinations of dirac impulses, i.e., $I^{\$}=\left\{\sum_{j=1}^{k} a_{j_{k}} \cdot \delta_{t_{k}} \mid a_{j_{k}} \in\right.$ $\left.\mathbf{K} \wedge t_{k} \in \mathbf{R}_{+}\right\}$. From a purely algebraic point of view, the only essential difference in the input signals between the discrete and continuous-time cases in this first approximation is that in the latter nonzero discrete values may occur at any time $t \geq 0$, while in the former case inputs may occur only at those time points indexed by integers. The truly interesting additional dimension, which makes the continuous-time case so much richer than its discrete-time counterpart, is that we may utilize the density of $I^{\$}$ in suitable spaces of $I$-valued distributions to yield a much richer space of input signals, including all of the traditional "smooth" inputs.

It is important to note that, with this model, $q(t)$ is only well-defined in the case that the input $i$, regarded as a distribution, can be truncated at time $t$. This representation is in agreement with those forwarded by Kalman and Hautus [KH] and Kamen [K3]. 
A simple example will help illustrate both the generality and the limitations of this framework. Consider a transmission line with series inductance $L$, series resistance $R$, shunt capacitance $C$, and shunt conductance $A$, all per unit length. We work with a unit length of such line, driven at $x=1$ and terminated at $x=0$ with a short circuit. We furthermore assume that the behavior of this line is governed by the standard wave equations, and that the loss is small enough that the characteristic impedance is given by $Z_{o}=\sqrt{L / C}$. See, e.g., [BSH]. Let $V(x, t)$ represent the voltage accross the line at position $x(0 \leq x \leq 1)$ at time $t$, and let $J(x, t)$ similarly represent the current on the line. The state space $Q$ is $\left\{(V(x), J(x)) \in \mathcal{C}_{c}^{\prime}(0,1] \times \mathcal{C}_{c}^{\prime}[0,1] \mid V(x)-Z_{o} \cdot J(x)=\right.$ 0 in some neighborhood of $x=1\}$. The requirement that the voltage be 0 at $x=0$ is recaptured by the fact that the voltage distribution be in $C_{c}^{\prime}(0,1]$, the space of all scalar-valued distributions with compact support contained in the half-open interval $(0,1] . C_{c}^{\prime}[0,1]$ is defined similarly, and $V(x)-Z_{o} \cdot J(x)=0$ in some neighborhood of 1 states that the impedance at $x=1$ be matched.

We drive the line with an impedance matched generator at $x=1$, so that $I$ takes values in the field $\mathbf{K}$, and the input over time has the form like $\left(\begin{array}{c}Z_{o} \\ -1\end{array}\right) \cdot i(t)$, with $i(t)$ a real-valued signal (represented as a distribution). (The current is negative since it is travelling to the left.) It is straightforward to show that the dynamics are represented by the following differential equation.

$$
\frac{d}{d t}\left(\begin{array}{c}
V(x, t) \\
I(x, t)
\end{array}\right)=\left(\begin{array}{cc}
-\frac{R}{L} & -\frac{1}{L} \cdot \frac{\partial}{\partial x} \\
-\frac{1}{C} \cdot \frac{\partial}{\partial x} & -\frac{A}{C}
\end{array}\right)\left(\begin{array}{c}
V(x, t) \\
J(x, t)
\end{array}\right)+\left(\begin{array}{c}
Z_{o} \\
-1
\end{array}\right) i(t)
$$

Now consider the output representation. We would like to observe the (natural) voltage-current pair at $x=1$. However, the state is a distribution, and not necessarily an ordinary function of time. Therefore, it does not make sense to sample the values at $x=1$ directly. There are two ways to address this issue. The first is to restrict the state distribution to a half-open neighborhood $(1-\mathfrak{l}, 1]$, where $\mathrm{l}$ is some small number. This is easily realized by using the natural surjections $\rho_{1}: C_{c}^{\prime}(0,1] \rightarrow C_{c}^{\prime}(1-\mathrm{l}, 1]$ and $\rho_{2}: C_{c}^{\prime}[0,1] \rightarrow C_{c}^{\prime}(1-\mathrm{l}, 1]$ (see [T]). In this case, the output space $Y$ is $C_{c}^{\prime}(1-\mathbf{l}, 1]$, and we get an output equation of the form

$$
y(t)=\left(\begin{array}{cc}
\rho_{1} & 0 \\
0 & \rho_{2}
\end{array}\right) q(t)
$$

The second approach is to regard the output space $Y$ as consisting of just pairs of real numbers $(V, I) \in \mathbf{K} \times \mathbf{K}$, representing values averaged about the point $x=1$. In this case, $h$ becomes an evaluation of the state distribution on a certain test function pair $\left(\varphi_{1}, \varphi_{2}\right) \in \mathcal{C}_{c}^{\prime}(1-\mathbf{l}, 1]^{2}$. Typically, this test function would have unit area. The output equation would then take the following form, with " $*$ " representing distributional convolution.

$$
y(t)=\left(\begin{array}{cc}
\varphi_{1} *(-) & 0 \\
0 & \varphi_{2} *(-)
\end{array}\right) q(t)
$$

The reader may find it instructive to compare this representation to that presented by Helton in [H6], in which he argues against requiring that $g$ and $h$ be continuous. 


\section{Behavior}

One of the cornerstones of the algebraic approach to discrete-time linear systems outlined in Section 1 is that is provides a natural bijective correspondence between canonical internal representations (represented by equations (4)) and input-output behaviors (represented by the $K[z]$-module homomorphism $\sigma \circ \rho$ ) in diagram (5). The approach presented here recaptures this natural corrrespondence completely within the context of continuous-time systems. In this paper, we focus upon the construction of the behavior from the internal representation, although we do disucss canonical realization briefly in the concluding section.

A key feature of our approach is that the notion of system is further parameterizable, both by choice of the underlying category $\mathcal{K}$ and by choice of the class of dynamics permitted. This, in turn, defines the exact nature of a typical behavior $\sigma \circ \rho$. The category $\mathcal{K}$ itself may be varied by regulating the degree of completeness of its objects; e.g., sequentially-complete spaces, quasi-complete spaces, and complete spaces. (The morphisms themselves are always taken to be continuous linear maps.) More importantly, however, the ring $\mathbf{K}[z]$ of the discrete-time case may be replaced by any member of a family of topological rings, which we term $\mathbf{R}_{+}$-rings. The table given below illustrates a few of the more important examples, which are elaborated in the next section. In each case, $I$ and $Y$ are locally convex spaces. The measures in $I^{\$}$ are $I$-valued measures (or distributions) in the sense of Schwartz [S2], and the outputs in $Y_{\$}$ are functions from the nonnegative reals into $Y$.

\begin{tabular}{|c|c|c|}
\hline Type of Dynamics & ${\text { Inputs in } I^{\$}}^{\text {Outputs in } Y_{\$}}$ \\
\hline \hline $\begin{array}{c}\text { locally equicontinuous } \\
\text { semigroups }\end{array}$ & $\begin{array}{c}\text { measures with } \\
\text { compact support }\end{array}$ & $\begin{array}{c}\text { continuous } \\
\text { functions }\end{array}$ \\
\hline $\begin{array}{c}\text { infinitely differentiable } \\
\text { semigroups }\end{array}$ & $\begin{array}{c}\text { distributions with } \\
\text { compact support }\end{array}$ & $\begin{array}{c}C^{\infty} \\
\text { functions }\end{array}$ \\
\hline $\begin{array}{c}\text { equicontinuous } \\
\text { semigroups }\end{array}$ & $\begin{array}{c}\text { uniform bounded } \\
\text { additive measures }\end{array}$ & $\begin{array}{c}\text { bounded and uniformly } \\
\text { continuous functions }\end{array}$ \\
\hline bounded & $L^{1}$ & $\begin{array}{c}\text { bounded continuous } \\
\text { functions }\end{array}$ \\
semigroups & measures & $\begin{array}{c}\text { continuous functions } \\
\text { which vanish at } \infty\end{array}$ \\
\hline $\begin{array}{c}\text { stable } \\
\text { semigroups }\end{array}$ & measures & $\begin{array}{c}\text { continuous functions with } \\
\text { scalar-compact suppport }\end{array}$ \\
\hline finite-response \\
semigroups
\end{tabular}

There is a fundamental measure/test-function duality between $I^{\$}$ and $Y_{\$}$, so that, in general, the more measures in $I^{\$}$, the fewer functions in $Y_{\$}$. There is no single choice of topological ring which provides a biggest version of each. Rather, the choice is a modelling problem; one of choosing the best framework for the systems being considered. Note also that bounded semigroups and stable semigroups take the same space of inputs. The distinction comes in that this space is topologized differently in each case, so that the underlying topological ring is not the same. 
Key to our approach is that the notion of a system may be viewed as being in either of two fundamental forms; its dynamics $(Q, \gamma)$ may be a (sufficiently continuous) module dynamics (in which $\gamma$ is a module homomorphism), or a semigroup dynamics (in which $\gamma$ is a $\left(C_{o}\right)$-semigroup of operators of a certain class. The correspondence is completely bidirectional; any topological module in the appropriate category uniquely defines a corresponding semigroup, and from any such semigroup we may recover the corresponding module. This association is crucial to the development of the theory, because while the semigroup model is much more natural from a system-theoretic point of view, the module approach provides the mathematical formulation necessary to establish the key results in a natural algebraic fashion.

\section{3. $\mathbf{R}_{+}$-Rings and the Representation of $\left(C_{o}\right)$ Semigroups}

Fundamental to our approach is that interesting classes of $\left(C_{o}\right)$ semigroups have natural representations as certain topological modules over $\mathbf{R}_{+}$-rings. In this section, the characterization of such representations is presented, and several important examples are provided. Due to their extremely technical functional-analytic nature, we have chosen to defer detailed development and proofs of many of the results to a separate work [H4]. Here we have attempted to emphasize the system-theoretic aspects.

We freely use concepts, terminology, and notation from the theory of locally convex spaces; we refer the reader [K6] and [K7] for details. Although we have eschewed the use of advanced category theory, we do occasionally employ some elementary terminology and concepts; the reader is directed to $[\mathrm{HS}]$ as a clarifying reference.

\section{Essential Background}

3.1 Notions Based Upon Precompact Subsets of an l.c.s. Throughout this work, 1.c.s. will serve as an abbreviation for locally convex topological vector space. Given an l.c.s. $E, \mathcal{U}(E)$ will denote the set of all balanced, closed, and convex neighborhoods of 0 , and $\mathcal{P}(E)$ will denote the set of all closed absolutely convex precompact subsets. Following [K6, §21,6, §21,7, §23,9], we define $E_{c}^{\prime}$ to be the dual of $E$ with the topology of uniform convergence on precompact subsets of $E$, and we let $E_{c}^{\prime \prime}$ be the corresponding bidual. Since we shall not consider other topologies on the dual in this work, these will frequently be abbreviated to $E^{\prime}$ and $E^{\prime \prime}$, respectively. If the natural injection $E \rightarrow E_{c}^{\prime \prime}$ is continuous (it will then be an embedding as well), then $E$ is termed almost polar reflexive. If it is surjective as well (hence an isomorphism), then $E$ is polar reflexive. Examples abound; every Fréchet (or (F)) space and, more generally, every strict (LF) space is polar reflexive. (See [H4].) In addition, we shall term an 1.c.s.a $(P F)$ space if it is the polar dual of a Fréchet space. Generally speaking, (PF) spaces enjoy the same properties relative to compact sets that (DF) spaces do relative to bounded sets.

The space $E$ is $c$-complete if every precompact subset is relatively compact. Every polar reflexive space is c-complete [K6, $23,9(1)]$. More generally, a subset $H$ of $E$ is $c$-dense in $E$ if every 
precompact $S \subseteq E$ is contained in the closure (in $E$ ) of $H \cap T$, for some precompact $T \subseteq E$. If $E$ is almost polar reflexive, then $E^{\prime \prime}$ is the c-completion of $E$.

If $E$ and $F$ are 1.c.s.'s, then $\mathbf{L}_{c}(E, F)$ denotes the space of all continuous linear mappings from $E$ to $F$ with the topology of uniform convergence on precompact subsets of $E$. $\mathbf{L}_{c}(E)$ denotes $\mathbf{L}_{c}(E, E) . \mathbf{L}(E, F)$ and $\mathbf{L}(E)$ denote the same spaces, but without any assigned topology.

A bilinear mapping $b: E \times F \rightarrow G$ on 1.c.s.'s is termed c-hypocontinuous if it is hypocontinuous with respect to the precompact subsets of $E$ and $F$. A c-hypocontinuous topological algebra is an 1.c.s. $R$ which is endowed with a c-hypocontinuous multiplication $*_{R}: R \times R \rightarrow R$ and a distinguished element 1 such that $\left(R,+, *_{R}\right)$ is a commutative ring with unit 1 . We shall always denote multiplication in such a ring $R$ by $*_{R}$, or by just $*$ if no confusion can result.

3.2 Theorem - Characterization of Precompactness in $\mathbf{L}_{c}(E, F)$ Let $E$ and $F$ be l.c.s.'s, and suppose that $E$ is almost polar reflexive. Then $H \subseteq \mathbf{L}_{c}(E, F)$ is precompact if and only if the following two conditions are satisfied.

(a) H is equicontinuous.

(b) $H(K)$ is precompact for each $K \in \mathcal{P}(E)$.

Proof: ((a), (b) $\Rightarrow H$ precompact) is a special case of Ascoli's Theorem [B4, Ch. X, §2.5, Thm. 2].

(H precompact $\Rightarrow(\mathrm{b})$ ) Let $K \in \mathcal{P}(E)$ and let $U \in \mathcal{U}(F)$. Since $H$ is precompact, there are $h_{1}, \ldots, h_{n} \in H$ such that given any $h \in H, h-h_{i} \in\{f \mid f(K) \subseteq U / 2\}$ for some $i \in[1, n]$. Trivially $\left\{h_{1}, \ldots, h_{n}\right\}$ is equicontinuous, so there is a $V \in \mathcal{U}(E)$ such that $h_{i}(V) \subseteq V / 2$ for all $i \in[1, n]$. Since $K$ is precompact, there are $k_{1}, \ldots, k_{m} \in K$ such that given any $k \in K, k-k_{i} \in V$ for some $i \in[1, m]$. Now let $x \in H(K)$; then $x=h(k)$ for some $h \in H$ and $k \in K$. By the preceding there are $h_{i}$ and $k_{j}$ such that $h-h_{i} \in\{f \mid f(K) \subseteq U / 2\}$ and $k-k_{j} \in V$. But then $h(k)-h_{i}\left(k_{j}\right)=$ $\left(h(k)-h_{i}(k)\right)+\left(h_{i}(k)-h_{i}\left(k_{j}\right)\right) \in U / 2+U / 2 \subseteq U$. Hence $\left\{h_{i}\left(k_{j}\right) \mid i \in[1, n] \wedge j \in[1, m]\right\} \subseteq H(K)$ is a finite set of points which are $U$-close to any $x \in H(K)$. Thus $H(K)$ is precompact.

( $H$ precompact $\Rightarrow$ (a)) Let $H \subseteq \mathbf{L}_{c}(E, F)$ be precompact; we must show that it is equicontinuous. First note that there is a natural embedding ' $: \mathbf{L}_{c}(E, F) \rightarrow \mathbf{L}_{\varepsilon}\left(F^{\prime}, E^{\prime}\right)$, where $\varepsilon$ defines the equicontinuous subsets of $F^{\prime}$. Since' ${ }^{\prime}$ is continuous, $H^{\prime} \subseteq \mathbf{L}_{\varepsilon}\left(F^{\prime}, E^{\prime}\right)$ is also precompact, and since $\varepsilon$ defines precompact sets [K6, §21,6(3)], Ascoli's Theorem may be invoked to deduce that $H^{\prime}(x)$ is precompact in $E^{\prime}$ for each $x \in F^{\prime}$. Also, by ( $H$ precompact $\left.\Rightarrow(b)\right)$ above, $H(K)$ is precompact for each $K \in \mathcal{P}(E)$, so $H^{\prime}$ is an equicontinuous subset of $\mathbf{L}\left(F^{\prime}, E^{\prime}\right)$. Hence we may apply Ascoli's Theorem again to deduce that $H^{\prime}$ is precompact in $\mathbf{L}_{c}\left(F^{\prime}, E^{\prime}\right)$. Now use $(H$ precompact $\Rightarrow(b))$ once again to ascertain that $H^{\prime}(K)$ is precompact for each $K \in \mathcal{P}\left(F^{\prime}\right)$. Finally, transpose again to get that $H^{\prime \prime}$ is equicontinuous in $\mathbf{L}\left(E^{\prime \prime}, F^{\prime \prime}\right)$. Since $E$ is almost polar reflexive, we have that $E \rightarrow E^{\prime \prime}$ is continuous, so $H_{\mid E}^{\prime \prime} \subseteq \mathbf{L}\left(E, F^{\prime \prime}\right)$ is also equicontinuous. Since each $h \in H^{\prime \prime}$ is the bitranspose of an element of $H, H^{\prime \prime}(E) \subseteq F$. The topology which $F^{\prime \prime}$ induces on $F$ is finer than the initial topology, so $H_{\mid E}^{\prime \prime}=H \subseteq \mathbf{L}(E, F)$ is also equicontinuous. 
3.3 Corollary - Characterization of c-Hypocontinuity Let E, F, $G$ be l.c.s.'s, and assume further that $E$ is almost polar reflexive. Let $b: E \times F \rightarrow G$ be a bilinear mapping which is hypocontinuous with respect to the precompact subsets of $E$ and the finite subsets of $F$. Then:

(a) b is c-hypocontinuous.

(b) $b\left(K_{1} \times K_{2}\right)$ is precompact for $K_{1} \in \mathcal{P}(E), K_{2} \in \mathcal{P}(F)$.

(c) The transpose $b_{1}: E \times G^{\prime} \rightarrow F^{\prime}$ (resp. $b_{2}: G^{\prime} \times F \rightarrow E^{\prime}$ is hypocontinuous with respect to the precompact subsets of $E$ (resp. $F$ ).

Proof: (a) In view of the hypotheses, we may define a continuous linear mapping $\bar{b}: F \rightarrow$ $\mathbf{L}_{c}(E, G)$ defined on elements by $x \mapsto b(-, x)$. For any $K \in \mathcal{P}(F), \bar{b}(K)$ is precompact, and so equicontinuous by the above theorem. But the equicontinuity of $\bar{b}(K)$ is equivalent to the hypocontinuity of $b$ with respect to the precompact subsets of $F$, as was to be proved.

(b) Argue as above, noting that by the above theorem, $\bar{b}(K)$ maps precompact sets into precompact sets.

(c) This is just the transpose of (b).

3.4 Completion Operators and Categories Recall that the full completion operator $\widehat{ }$ on an 1.c.s. provides, for each complete 1.c.s. $F$ and morphism $f: E \rightarrow F$, a unique extension $\widehat{f}: \widehat{E} \rightarrow F$ such that $f=\widehat{f} \circ i$, with $i: E \rightarrow \widehat{E}$ the natural embedding. This construction is generalized as follows. Let $\sim$ be an operator which defines subsets of 1.c.s.'s such that for any continuous linear mapping $f: E \rightarrow F$ of 1.c.s.'s, $S \in^{\sim}(E)$ implies that $f(S) \in^{\sim}(F)$. For example, $\sim$ may define all bounded subsets, all precompact subsets, or just all subsets. Call an 1.c.s. $E{ }^{\sim}$-complete if the closure of each $S \in \sim^{\sim}(E)$ is complete. The ${ }^{\sim}$-completion $\widetilde{E}$ (or $(E)^{\sim}$ ) of an 1.c.s. $E$ is the intersection of all subspaces $F$ of $\widetilde{E}$ such that $E \subseteq F$ and $F$ is ${ }^{\sim}$-complete. As $\widetilde{E}$ is surely complete, there is at least one such $F$. Furthermore, if $S \in \sim(E)$, then $S \in \sim(F)$ for all ${ }^{\sim}$-complete $F$ with $E \subseteq F$; hence the closure of $S$ is in $\sim(\widetilde{E})$ and is complete, so $\widetilde{E}$ is $\sim$-complete. On the

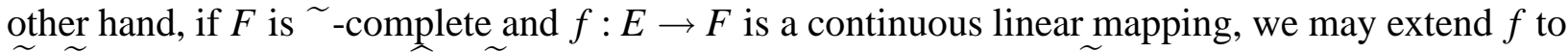
$\widetilde{f}: \widetilde{E} \rightarrow F$ by restricting $\widehat{f}$ to $\widetilde{E}$. It is straightforward to verify that $\widetilde{f}$ is the unique morphism such that $f=i \circ \widetilde{f}$, with $i: E \rightarrow \widetilde{E}$ the natural embedding. An operator ${ }^{\sim}$ meeting the above conditions is called a partial completion operator. Some particular examples to keep in mind are the following.

(a) All subsets; this yields just the full-completion operator, denoted $\widehat{ }$.

(b) All bounded subsets; this yields the quasi-completion operator.

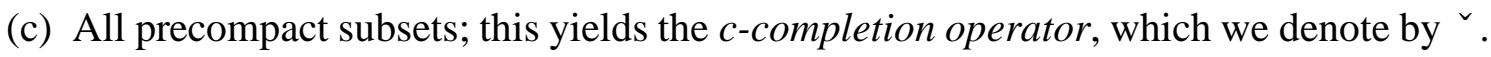

(d) All Cauchy sequences; this yields the sequential-completion operator.

(e) All finite sets; this yields the identity operator.

LCS denotes the category of all 1.c.s.'s with continuous linear maps as morphisms, and for any partial completion operator $\sim \widetilde{\mathbf{L C S}}$ denotes the full subcategory of LCS whose objects are the 〜-complete 1.c.s.'s. 
Occasionally, it will be useful to compare partial completion operators. We write $\sim_{1} \leq \sim_{2}$ if every space which is ${ }_{2}$-complete is also ${ }_{1}$-complete. In the above examples, we have $(\mathrm{e}) \leq$ (d) $\leq(\mathrm{c}) \leq(\mathrm{b}) \leq(\mathrm{a})$.

\section{Semigroup Representation by Modules}

3.5 $\mathbf{R}_{+}$-Rings It is easy to see that, under addition, the nonnegative reals $\mathbf{R}_{+}$form a commutative topological monoid with identity 0 . An $\mathbf{R}_{+}$-ring is a pair $(R, i)$ in which $R$ is a chypocontinuous topological algebra and $i: \mathbf{R}_{+} \rightarrow R$ is a continuous mapping subject to the following conditions.

(r1) $i$ is a monoid homomorphism for the additive structure of $\mathbf{R}_{+}$and the multiplicative structure of $R$.

(r2) $\operatorname{span}\left(i\left(\mathbf{R}_{+}\right)\right)$is c-dense in $R$.

(r3) $R$ is almost polar reflexive.

(r4) $R^{\prime}$ is complete.

The $\mathbf{R}_{+}$-ring $(R, i)$ will often be abbreviated to just $R$ when $i$ is clear from context or irrelevant. For any $\mathbf{R}_{+}$-ring $(R, i), \operatorname{span}\left(i\left(\mathbf{R}_{+}\right)\right)$forms a subring of $R$. We shall denote this subring by $\Delta(R)$, and term it the skeleton of $R$. $i(t)$ is often denoted by $\delta_{t}$, since it turns out to be the Dirac delta centered at $t$ in most examples which we consider. In view of (r2), we may always extend the hypocontinuity of $*_{\Delta(R)}$ and $*_{R}$ to $\check{R}$. Thus, $\widetilde{\Delta(R)}$ and $\widetilde{R}$ are $\mathbf{R}_{+}$-rings for any partial completion operator ${ }^{\sim}$ which defines precompact sets. It is furthermore the case that $(\Delta(R))^{\vee}=\check{R}$, and this space is polar reflexive. It is not necessarily the case that $\check{R}=\widehat{R}$; however, in most examples which we consider, $R^{\prime}$ will be a strict (LF) space, and hence both complete (yielding (r4)) and bornological (guaranteeing that $R^{\prime \prime}=\check{R}$ is complete).

A morphism $f:(R, i) \rightarrow(S, j)$ of $\mathbf{R}_{+}$-rings is a continuous linear mapping $f: R \rightarrow S$ such that $f \circ i=j$. The density of $\Delta(R)$ in $R$ ensures that a morphism of $\mathbf{R}_{+}$-rings is a ring homomorphism in the usual sense. In fact, there can be at most one morphism from $(R, i)$ to $(S, j)$, as the value of any $f:(R, i) \rightarrow(S, j)$ is determined by its values on $\Delta(R)$, and $f\left(\delta_{t}\right)=\delta_{t}$.

3.6 $(R)$-Modules Given an $\mathbf{R}_{+}$-ring $(R)$, an $(R)$-module is an 1.c.s. $E$ together with a bilinear action $b: R \times E \rightarrow E$ which is c-hypocontinuous and which satisfies the usual module axioms. A morphism $f:(E, b) \rightarrow(F, c)$ of $(R)$-modules is just a continuous linear map which is also a module homomorphism.

Note: We shall always use the term $(R)$-module (with parentheses around the $R$ ) for a chypocontinuous module action, while $R$-module shall mean just a module over the (ordinary) ring $R$. 
3.7 $(R)$-Semigroups We use the definition of Kōmura [K5] in defining a locally equicontinuous semigroup $(E, T)$ as an $\mathbf{R}_{+}$-indexed family $T: \mathbf{R}_{+} \rightarrow L(E)$ of operators on the 1.c.s. $E$. This specifically mandates, in addition to the semigroup axioms and the equicontinuity of $\{T(t) \mid 0 \leq t \leq$ $\varepsilon\}$ for any $\varepsilon \geq 0$, that $\lim _{t \rightarrow 0} T(t) e=e$ for any $e \in E$. For the sake of brevity, we shall refer to such semigroups of operators as just semigroups. We let $\mathbf{g}_{T}$ denote the infinitesimal generator of such a semigroup. If $E$ is sequentially complete, then $\mathbf{g}_{T}$ is densely defined and uniquely determines $T$ [K5].

A morphism $f:(E, T) \rightarrow(F, S)$ of semigroups is a continuous linear mapping $f: E \rightarrow F$ such that, for each $t \in \mathbf{R}_{+}, f \circ T(t)=S(t) \circ f$.

Let $R$ be an $\mathbf{R}_{+}$-ring, and let $(E, b)$, be an $(R)$-module. Define the mapping $\mathbf{T}_{b}: \mathbf{R}_{+} \rightarrow L(E)$ by $\mathbf{T}_{b}=\left(e \mapsto b\left(\delta_{t}, e\right)\right)$. We call $\mathbf{T}_{b}$ the semigroup associated with $(E, b)$, and any semigroup arising in this fashion an $(R)$-semigroup. This terminology is justified by the following proposition.

3.8 Proposition Let $(R, i)$ be an $\mathbf{R}_{+}$-ring, and let $(E, b)$ be an $(R)$-module. Then $\mathbf{T}_{b}$ is a semigroup, and, furthermore, if either $E$ or $R$ is sequentially complete, then $\mathbf{g}_{\mathbf{T}_{b}}$ is densely defined and so uniquely determines $\mathbf{T}_{b}$.

PROOF: Consult [H4].

We now turn to the converse characterization; namely, that each $(R)$-semigroup determines a unique $(R)$-module. Given a semigroup $(E, T)$, define its natural response $\mathbf{r}_{T}: E \rightarrow \mathcal{C}\left(\mathbf{R}_{+}, E\right)$ by $e \mapsto(t \mapsto T(t) e)$. Here $C\left(\mathbf{R}_{+}, E\right)$ is the space of all continuous functions from $\mathbf{R}_{+}$to $E$, endowed with the topology of uniform convergence on compact subsets of $\mathbf{R}_{+}$. It is easy to verify that $\mathbf{r}_{T}$ is continuous. Define the mapping $\mathbf{I}(R, E): \mathbf{L}_{c}(R, E) \rightarrow \mathcal{C}\left(\mathbf{R}_{+}, E\right)$ by $f \mapsto\left(t \mapsto f\left(\delta_{t}\right)\right)$. We then have the following.

3.9 (R)-Semigroup Characterization Theorem Let $(E, T)$ be a semigroup and let $R$ be an $\mathbf{R}_{+}$-ring. Then $(E, T)$ is an $(R)$-semigroup if and only if there is a unique continuous linear mapping $\vee: E \rightarrow \mathbf{L}_{c}(R, E)$ such that the following diagram commutes.

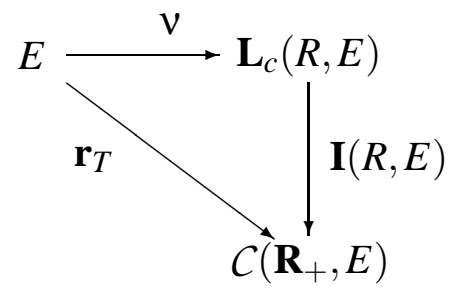

PROOF: Consult [H4]. 
In other words, a semigroup $(E, T)$ is an $(R)$-semigroup if and only if its natural response may be interpreted as an element of $\mathbf{L}_{c}(R, E)$, when this space is regarded (via $\mathbf{I}(R, E)$ ) as a subspace of $C\left(\mathbf{R}_{+}, E\right)$ ). Let $\mathbf{b}_{T, R}: R \times E \rightarrow E$ denote the bilinear mapping corresponding to $\vee: E \rightarrow \mathbf{L}(R, E)$ in the above diagram. It is easy to see that $\mathbf{b}_{T, R}$ recovers precisely the $(R)$-module action corresponding to the semigroup $(E, T)$; thus we call $\mathbf{b}_{T, R}$ the $(R)$-module associated with $(E, T)$. This completes our characterization of the natural correspondence between $(R)$-semigroups and $(R)$ modules. To emphasize this, we state it as a formal result.

3.10 Theorem - Natural Correspondence Let $R$ be an $\mathbf{R}_{+}$-ring. operator. Then the $(R)$ semigroups and the $(R)$-modules are in a natural correspondence, characterized by the following.

(a) Let $(E, T)$ be an $(R)$-semigroup. Then $\mathbf{T}_{\mathbf{b}_{T, R}}=T$.

(b) Let $b: R \times E \rightarrow E$ be an $(R)$-module. Then $\mathbf{b}_{\mathbf{T}_{b}, R}=b$.

(c) An $h \in \mathbf{L}(E, F)$ is a morphism of $(R)$-semigroups $h:(E, T) \rightarrow(F, S)$ if and only if it is a morphism of the corresponding $(R)$-modules $h:\left(E, \mathbf{b}_{T, R}\right) \rightarrow\left(F, \mathbf{b}_{S, R}\right)$.

Categorically speaking, if we fix a category $\mathcal{K}$ of 1.c.s.'s, and then define $\mathbf{C S G}(R, \mathcal{K})$ to be the category of all $(R)$-semigroups over spaces in $\mathcal{K}$, with morphisms precisely the $(R)$-semigroup morphisms whose underlying functions are $\mathcal{K}$-morphisms, and if we define the category of $(R)$ modules $\operatorname{MOD}(R, \mathcal{K})$ similarly, then the above result says precisely that these two categories are naturally equivalent.

3.11 More on the Issue of Completeness It is well known that to ensure a unique solution to the differential equation (6), the map $f$, which is taken to be the infinitesimal generator of a semigroup $(Q, T)$, must be densely defined [K5, Thm. 3]. We know by 3.8 that if $(Q, T)$ is an $(R)$ semigroup for any sequentially complete $\mathbf{R}_{+}$-ring $R$, or if $Q$ itself is sequentially complete, then this will be the case. In particular, if $Q$ is sequentially complete, then the $(R)$-semigroups and $(\Delta(R))$ semigroups coincide, and each such semigroup will have a densely defined infinitesimal generator. Unfortunately, there does not seem to be a nice algebraic way to characterize exactly those $(\Delta(R))$ semigroups which have densely defined infinitesimal generators, unless the underlying spaces are sequentially complete. This is apparently a consequence of the fact that having a densely defined infinitesimal generator is not an "algebraic" property of a semigroup. To get aggregate completion of a class of semigroups, we must mandate sequential completeness of either the underlying $\mathbf{R}_{+}$ring or else the underlying space of each semigroup. For more on this issue, consult [H4].

\section{Example Generation}

So far, our presentation has been completely abstract. We now turn to the issue of explicitly identifying ways of producing $\mathbf{R}_{+}$-rings. In view of 3.9, to characterize the natural responses of $(R)$-semigroups, it suffices to provide a characterization of $\mathbf{L}_{c}(R, E)$. We next provide a way to explicitly produce the desired $\mathbf{R}_{+}$-ring, based upon this set of responses. 
3.12 Definition Let $\mathcal{F}$ denote an operator which assigns to each 1.c.s. $E$ a corresponding 1.c.s. $\mathcal{F}\left(\mathbf{R}_{+}, E\right)$ of continuous functions from $\mathbf{R}_{+}$to $E$. When $E=\mathbf{K}$, we write $\mathcal{F}\left(\mathbf{R}_{+}, E\right)$ as $\mathcal{F}\left(\mathbf{R}_{+}\right)$. $\mathcal{F}$ is called an $\mathbf{R}_{+}$-ring generator if it satisfies the following conditions.

(g1) For each $t \in \mathbf{R}_{+}$, the point measure $\left(\delta_{t}: \varphi \mapsto \varphi(t)\right) \in \mathcal{F}^{\prime}\left(\mathbf{R}_{+}\right)$.

(g2) For each 1.c.s. $E$ and $t \in \mathbf{R}_{+}, \mathcal{F}\left(\mathbf{R}_{+}, E\right)$ is closed under the left translation operator $\tau_{t}: f \mapsto f(\cdot+t)$, and the translation inclusion $\mathcal{F}\left(\mathbf{R}_{+}\right) \rightarrow \mathcal{F}\left(\mathbf{R}_{+}, \mathcal{F}\left(\mathbf{R}_{+}\right)\right)$given by $\varphi \mapsto\left(t \mapsto \tau_{t}(\varphi)\right)$ is continuous.

(g3) There is a natural isomorphism $\mathcal{F}\left(\mathbf{R}_{+}, E\right) \cong \mathbf{L}_{c}\left(\Delta\left(\mathcal{F}^{\prime}\left(\mathbf{R}_{+}\right)\right), E\right)$ given by $f \mapsto\left(\delta_{t} \mapsto\right.$ $f(t))$, where $\Delta\left(\mathcal{F}^{\prime}\left(\mathbf{R}_{+}\right)\right)$is defined to be the subspace of $\mathcal{F}^{\prime}\left(\mathbf{R}_{+}\right)$spanned by $\left\{\boldsymbol{\delta}_{t} \mid t \in\right.$ $\left.\mathbf{R}_{+}\right\}$with the induced topology.

(g4) $\mathcal{F}\left(\mathbf{R}_{+}\right)$is complete.

3.13 Theorem Let $\mathcal{F}$ be an $\mathbf{R}_{+}$-ring generator. Then $\mathcal{F}^{\prime}\left(\mathbf{R}_{+}\right)$is an $\mathbf{R}_{+}$-ring with multiplication convolution of measures on the functions in $\mathcal{F}\left(\mathbf{R}_{+}\right)$. Furthermore, $(E, T)$ is a $\left(\Delta\left(\mathcal{F}^{\prime}\left(\mathbf{R}_{+}\right)\right)\right)$semigroup if and only if its response $\mathbf{r}_{T}$ lies in $\mathcal{F}\left(\mathbf{R}_{+}, E\right)$.

PROOF: Consult [H4].

Whenever we extract the $\mathbf{R}_{+}$ring $\mathcal{F}^{\prime}\left(\mathbf{R}_{+}\right)$from the $\mathbf{R}_{+}$-ring generator $\mathcal{F}$, we shall always assume that the multiplication is convolution of measures.

In [S1], Laurent Schwartz defined a wide class of spaces of $m$-times differentiable functions, which he termed $\mathbf{H}^{m}$ spaces. From these, he defined, for an arbitrary l.c.s. $E$, the spaces $\widetilde{\mathbf{H}}^{m}(E)$, which consist of precisely those functions $f \in \mathcal{C}\left(\mathbf{R}_{+}, E\right)$ which have the property that $t \mapsto\left\langle f(t), e^{\prime}\right\rangle \in$ $\mathbf{H}^{m}$ for each $e^{\prime} \in E^{\prime}$, endowed with the topology of uniform convergence on equicontinuous subsets of $E^{\prime} .^{\dagger}$ He then proceeded to provide a wealth of examples. We may draw upon this wealth, as shown by the following.

3.14 Theorem For $m=0$ or $m=\infty$, every $\widetilde{\mathbf{H}}^{m}$ space in the sense of Schwartz such that $\mathcal{F}\left(\mathbf{R}_{+}\right)$ is complete and polar reflexive defines an $\mathbf{R}_{+}$-ring generator.

PROOF: Consult [H4].

Requiring $\mathcal{F}\left(\mathbf{R}_{+}\right)$to be complete and polar reflexive is not very constraining, and is satisfied by all reasonable examples of $\widetilde{\mathbf{H}}^{m}$, including those cases for which $\mathcal{F}\left(\mathbf{R}_{+}\right)$is a Fréchet space or, more generally, a strict (LF) space. However, the framework of Schwartz admits some rather anomalous examples which do not meet this criterion. See [H4] for a more complete discussion.

\footnotetext{
${ }^{\dagger}$ We only consider those spaces which satisfy hypotheses $\left(H_{m, m+l}\right)$ and $\left(H_{5}\right)$ as well as $\left(H_{1}\right)$ through $\left(H_{4}\right)$, of the framework of [S1]. In addition, Schwartz actually worked with the domain $\mathbf{R}^{n}$ rather than $\mathbf{R}_{+}$, but his results all easily translate to our framework. To avoid undue pedantry, we shall refer to his work as though he used $\mathbf{R}_{+}$.
} 


\section{Specific Examples}

We now proceed to give a number of specific examples. In general, we attempt to give systemtheoretic insight into the properties of each example, but omit proofs. In all cases, elaboration, with complete proof, may be found in [H4], even when not explicitly cited.

3.15 Example - All Semigroups The space $\mathcal{C}\left(\mathbf{R}_{+}, E\right)$ defines an $\mathbf{R}_{+}$-ring generator. It is one of the $\widetilde{\mathbf{H}}^{m}(E)$ spaces of Schwartz, and $\mathcal{C}\left(\mathbf{R}_{+}\right)$is a Fréchet space, so it is an $\mathbf{R}_{+}$-ring generator. It is also the most general of such generators, in that every semigroup is an $\Delta\left(\mathbf{R}_{+}\right)$-semigroup. Indeed, for any $\left(C^{\prime}\left(\mathbf{R}_{+}\right)\right)$-semigroup $(E, T)$, since $C\left(\mathbf{R}_{+}, E\right) \cong \mathbf{L}_{c}\left(\Delta\left(C^{\prime}\left(\mathbf{R}_{+}\right)\right), E\right), \mathbf{I}(R, E)$ must be the identity, up to isomorphism. Thus, in the notation of $3.9, \mathrm{v}=\mathbf{r}_{T}$; i.e., $(E, T)$ is a semigroup. $C^{\prime}\left(\mathbf{R}_{+}\right)$is a Fréchet space and consists of all measures on $\mathbf{R}_{+}$with compact support, in the sense of Bourbaki [B3].

Yamamoto [Y, Thm. 3.24] has shown that every semigroup $(E, T)$ on a complete l.c.s. admits the structure of a $C^{\prime}\left(\mathbf{R}_{+}\right)$-module which is hypocontinuous with respect to the precompact subsets of $C^{\prime}\left(\mathbf{R}_{+}\right)$and the finite subsets of $E$. He uses this result to construct a module action for a dynamics described by a semigroup, in much the same way as we have. On the other hand, he does not specifically address the converse question of constructing a semigroup from a module action, so he does not need to postulate c-hypocontinuity with respect to the precompact subsets of $E$, as we have.

3.16 Note - Finite Nonzero Differentiability It is tempting to propose that $C^{m}\left(\mathbf{R}_{+}, E\right)$, the space of all functions $f: \mathbf{R}_{+} \rightarrow E$ which are m-times differentiable, is an $\mathbf{R}_{+}$-ring generator. However, the associated ring action of convolution is not everywhere defined. For example, if $m=$ 1 , then $\delta^{\prime}(0) * \delta^{\prime}(0)=\delta^{\prime \prime}(0)$, which is in $C^{2}\left(\mathbf{R}_{+}\right)$, but not in $C^{1}\left(\mathbf{R}_{+}\right)$. This is why the requirement of $m=0$ or $m=\infty$ is necessary. For $m=\infty$ we have the following.

3.17 Example - Infinitely Differentiable Semigroups Let $\mathcal{E}\left(\mathbf{R}_{+}, E\right)$ denote the space of all infinitely differentiable functions $\mathbf{R}_{+} \rightarrow E$, with the topology of uniform convergence of functions and all derivatives on compact subsets of $\mathbf{R}_{+}$. The space $\mathcal{E}\left(\mathbf{R}_{+}, E\right)$ defines an $\mathbf{R}_{+}$-ring generator, since it is one of the $\widetilde{\mathbf{H}}^{m}(E)$ spaces of Schwartz and $\mathcal{E}\left(\mathbf{R}_{+}\right)$is a Fréchet space as well. It characterizes those responses which are infinitely differentiable, which Waelbroeck [W] has termed simply differentiable. $\mathcal{E}^{\prime}\left(\mathbf{R}_{+}\right)$is a (PF) space which consists of all distributions on $\mathbf{R}_{+}$with compact support, in the sense of Schwartz [S3]. This class of dynamics has several interesting properties, including that its semigroups have continuous infinitesimal generators, and that $\mathcal{E}\left(\mathbf{R}_{+}\right)$is reflexive, so that the strong dual and polar dual coincide. For these and other reasons, it has been used as the basis of several algebraic approaches to continuous-time linear systems, including [BDM], [K2],[KH], and [H2]. 
3.18 Example - Equicontinuous Semigroups A semigroup $(E, T)$ is equicontinuous if the entire family of operators $\left\{T(t) \mid t \in \mathbf{R}_{+}\right\}$is an equicontinuous subset of $\mathbf{L}(E)$. To characterize the responses of such semigroups, let $\mathcal{B}_{v}\left(\mathbf{R}_{+}, E\right)$ denote the space of all functions in $\mathcal{C}\left(\mathbf{R}_{+}, E\right)$ which are bounded and uniformly continuous. Endow this space with the topology of uniform convergence on $\mathbf{R}_{+}$; that is, with the topology defined by seminorms of the form $f \mapsto \sup _{t \in \mathbf{R}_{+}}|f(t)| . \mathcal{B}_{v}$ is an $\mathbf{R}_{+}$-ring generator, and we furthermore have the following characterization.

\subsection{Proposition Let $(E, T)$ be a semigroup. Then the following are equivalent.}

(a) $(E, T)$ is an equicontinuous semigroup of operators.

(b) For each $e \in E$, the function $\mathbf{r}_{T}(e)$ is uniformly continuous.

(c) $(E, T)$ is an $\left(\Delta\left(\mathcal{B}_{v}^{\prime}\left(\mathbf{R}_{+}\right)\right)\right)$-semigroup.

PROOF: Consult [H4].

$\mathcal{B}_{v}\left(\mathbf{R}_{+}, E\right)$ is not an $\widetilde{\mathbf{H}}^{m}(E)$ space in the sense of Schwartz, so that the class of spaces admissible as $\mathbf{R}_{+}$-ring generators extends beyond the class of $\widetilde{\mathbf{H}}^{m}(E)$ spaces.

$\mathcal{B}_{v}\left(\mathbf{R}_{+}\right)$is a Banach space with the supremum norm, but the space $\mathcal{B}_{v}^{\prime}\left(\mathbf{R}_{+}\right)$is not a Banach space under the polar topology. Rather, it is a (PF) space whose compact sets are the unit balls of the strong (Banach) dual. It consists of a special subspace of the space of all regular bounded additive measures [DS] on $\mathbf{R}_{+}$, which we term the uniform bounded additive measures. These "measures" are extremely general, and do not possess the countable additivity usually associated with measures. The reader is referred to [H4] for further description.

It is interesting to note that in equicontinuous semigroups the convergence is actually uniform on bounded sets. Indeed every equicontinuous subset of $\mathbf{L}(E)$ is bounded in $\mathbf{L}_{b}(E)$ (the topology of uniform convergence on bounded sets) $[\mathrm{K} 7, \S 39,3(1)]$.

3.20 Example - Stable Semigroups A common form of stability mandates that the natural response decay to 0 as time approaches infinity. To recapture this in terms of semigroups, call a semigroup $(E, T)$ stable if $\lim _{t \rightarrow \infty} T(t) e=0$ for each $e \in E$. To characterize such semigroups as module actions, for any l.c.s. $E$, let $\mathcal{C}_{o}\left(\mathbf{R}_{+}, E\right)$ denote the (topological) subspace of $\mathcal{B}_{v}\left(\mathbf{R}_{+}, E\right)$ consisting of those functions $f: \mathbf{R}_{+} \rightarrow E$ such that $\lim _{t \rightarrow \infty} f(t)=0$. The (PF) space $C_{o}^{\prime}\left(\mathbf{R}_{+}\right)$is an $\mathbf{R}_{+}$-ring generator, and the uniformly stable semigroups are then precisely the $\Delta\left(\left(C_{o}^{\prime}\left(\mathbf{R}_{+}\right)\right)\right)$semigroups. $C_{o}^{\prime}\left(\mathbf{R}_{+}\right)$is the space of all integrable (i.e., $L^{1}$ ) measures. (See, e.g., [H7, Chap. 4, $\S 5])$. Like $\mathcal{B}_{v}\left(\mathbf{R}_{+}, E\right), \mathcal{C}_{o}\left(\mathbf{R}_{+}, E\right)$ is not an $\widetilde{\mathbf{H}}^{m}(E)$ space in the sense of Schwartz.

3.21 Example - Bounded Semigroups A substantially weaker form of system theoretic stability than that discussed in the previous parargraph postulates that the response does not continue to grow indefinitely. If the underlying space $E$ of a semigroup is a Banach space, this is often formalized by stating that $\sup _{t \in \mathbf{R}_{+}}\|T(t) e\|$ is finite for each $e \in E$. In our more general context, call a semigroup $(E, T)$ bounded if, for each $e \in E,\left\{T(t) e \mid t \in \mathbf{R}_{+}\right\}$is a bounded subset of $E$. In 
an attempt to represent such semigroups, let $\mathcal{B}\left(\mathbf{R}_{+}, E\right)$ denote the space of all bounded functions in $\mathcal{C}\left(\mathbf{R}_{+}, E\right)$ topologized by seminorms of same form as those of $\mathcal{B}_{v}\left(\mathbf{R}_{+}, E\right) \cdot \mathcal{B}\left(\mathbf{R}_{+}\right)$is a Banach space; unfortunately $\lim _{t \rightarrow 0} \delta_{t} \neq \delta_{0}$ in $\mathcal{B}^{\prime}\left(\mathbf{R}_{+}\right)$, so it cannot be an $\mathbf{R}_{+}$-ring. Indeed, the function $t \mapsto \sin \left(t^{2}\right)$ is surely in $\mathcal{B}\left(\mathbf{R}_{+}\right)$, yet $\sin \left((t+\varepsilon)^{2}\right) \nrightarrow \rightarrow \sin \left(t^{2}\right)$ as $\varepsilon \rightarrow 0$ uniformly over all $t \in \mathbf{R}_{+}$. In other words, $\lim _{\varepsilon \rightarrow 0} \delta_{\varepsilon} * \sin \left(t^{2}\right) \neq \sin \left(t^{2}\right)$. From this it is easy to show that distributional convolution on $\mathcal{B}^{\prime}\left(\mathbf{R}_{+}\right)$is not c-hypocontinuous. This is why we use uniformly continuous functions in the above example; the translation of uniformly continuous functions is continuous under the supremum-norm topology.

To recapture the bounded semigroups, we endow $\mathcal{B}\left(\mathbf{R}_{+}\right)$with another topology, weaker and rather more complex. Define $L_{1 \downarrow}^{1}$ denote the set of all strictly positive and nonincreasing $L^{1}$ functions in $\mathcal{C}\left(\mathbf{R}_{+}\right)$. For each such function $\zeta$, define the space $\mathcal{B}\left(\left[\mathbf{R}_{+}, \zeta\right], E\right)$ to have as members all $f \in \mathcal{C}\left(\mathbf{R}_{+}, E\right)$ with the property that $\left\{\zeta(t) \cdot f(t) \mid t \in \mathbf{R}_{+}\right\}$is bounded in $E$, topologized by seminorms of the form $f \mapsto \sup _{t \in \mathbf{R}_{+}} \zeta(t) \cdot \alpha(f(t))$, with $\alpha$ a continuous seminorm on $E$. Clearly $\mathcal{B}\left(\mathbf{R}_{+}, E\right) \subseteq \mathcal{B}\left(\left[\mathbf{R}_{+}, \zeta\right], E\right)$; we let $\iota_{\zeta}: \mathcal{B}\left(\mathbf{R}_{+}, E\right) \rightarrow \mathcal{B}\left(\left[\mathbf{R}_{+}, \zeta\right], E\right)$ denote the continuous natural inclusion. Furthermore, if $\zeta_{1}(t) \geq \zeta_{2}(t)$ for all $t \in \mathbf{R}_{+}$, then we have a continuous natural inclusion $\mathfrak{\zeta}_{\zeta_{1}}: \mathcal{B}\left(\left[\mathbf{R}_{+}, \zeta_{1}\right], E\right) \rightarrow \mathcal{B}\left(\left[\mathbf{R}_{+}, \zeta_{2}\right], E\right)$. This yields a directed system, and we assign to $\mathcal{B}\left(\mathbf{R}_{+}, E\right)$ the projective limit topology $\lim _{\leftarrow} \zeta_{\alpha} \zeta_{\beta}\left(\mathcal{B}\left(\left[\mathbf{R}_{+}, \zeta_{\beta}\right], E\right)\right.$, which we denote by $\mathcal{B}_{\leftarrow}\left(\mathbf{R}_{+}, E\right)$. $\mathcal{B}_{\leftarrow}\left(\mathbf{R}_{+}, E\right)$ is the same space algebraically as $\mathcal{B}\left(\mathbf{R}_{+}, E\right)$, but its topology is stricly weaker than that of the latter. It is not a (PF) space. Also, $\mathcal{B}_{\leftarrow}^{\prime}\left(\mathbf{R}_{+}\right)$is the same space algebraically as $C_{o}^{\prime}\left(\mathbf{R}_{+}\right)$, but its topology is strictly stronger, since it has a larger dual. Nonetheless, $\mathcal{B}_{\leftarrow}$ is indeed an $\mathbf{R}_{+}{ }^{-}$ ring generator, and the $\left(\Delta\left(\mathcal{B}_{\leftarrow}^{\prime}\left(\mathbf{R}_{+}\right)\right)\right)$-semigroups are precisely the bounded ones. This shows strikingly that the same algebraic ring may underly more than one distinct $\mathbf{R}_{+}$-ring.

We note that $\mathcal{B}_{\leftarrow}\left(\mathbf{R}_{+}, E\right)$ is indeed an $\widetilde{\mathbf{H}}^{m}(E)$ space, which Schwartz [S1] denotes by $\mathbf{B}_{c}^{0}(E)$. However, we have constructed this space in quite a different fashion than did he.

A semigroup $(E, T)$ with $E$ barrelled is a bounded semigroup if and only if it is equicontinuous. Indeed, if $E$ is barrelled, then every simply bounded subset of $\mathbf{L}(E)$ is equicontinuous [K7, $\S 39,3(2)]$, hence, by 3.19 , every bounded semigroup on $E$ is equicontinuous, and hence its response is in fact in $\mathcal{B}_{v}\left(\mathbf{R}_{+}, E\right)$. Since Banach and Fréchet spaces, as well as (LF) spaces, are always barrelled, in most interesting examples a bounded response translates into equicontinuity. Nonetheless, it is possible to construct a bounded semigroup whose response is not uniformly continuous, so boundedness does not automatically imply uniform continuity for semigroups.

3.22 Finite-Response Semigroups Utilizing $C\left(\mathbf{R}_{+}, E\right)$ as the space of outputs yields all $\left(C_{o}\right)$ semigroups, as shown in 3.15. By restricting the outputs to be in $\mathcal{B}_{\leftarrow}\left(\mathbf{R}_{+}, E\right)$, in $\mathcal{B}_{v}\left(\mathbf{R}_{+}, E\right)$ and then in $\mathcal{C}_{o}\left(\mathbf{R}_{+}, E\right)$, we obtained successively more restricted classes of semigroup responses. Continuing to the extreme, we may consider only those elements of $\mathcal{C}\left(\mathbf{R}_{+}, E\right)$ which have compact support; that is, only those $f: \mathbf{R}_{+} \rightarrow E$ which vanish for all $t$ greater than some $t_{f}$. This is the space $\mathcal{C}_{\boldsymbol{K}}\left(\mathbf{R}_{+}, E\right)$, denoted $\mathbf{D}^{0}(E)$ in [S1]. Unfortunately, this choice does not generate an $\mathbf{R}_{+}-$ ring. Rather, we must work with the larger space $\mathcal{C}_{\sigma \kappa}\left(\mathbf{R}_{+}, E\right)$ of all functions in $\mathcal{C}\left(\mathbf{R}_{+}, E\right)$ with scalar-compact support. This space is an $\widetilde{\mathbf{H}}^{m}(E)$ space (denoted $\widetilde{\mathbf{D}}^{0}(E)$ in [S1]), and $C_{\sigma \kappa}^{\prime}\left(\mathbf{R}_{+}\right)$is complete, so it is an $\mathbf{R}_{+}$-ring generator. A continuous function $f: \mathbf{R}_{+} \rightarrow E$ is in $\mathcal{C}_{\sigma \kappa}\left(\mathbf{R}_{+}, E\right)$ pre- 
cisely in the case that, for each $e^{\prime} \in E^{\prime}$, the $\mathbf{K}$-valued function $t \mapsto\left\langle f(t), e^{\prime}\right\rangle$ has compact support. The topology on this space is defined as follows. On the space $\mathcal{C}_{\sigma \kappa}\left(\mathbf{R}_{+}\right)$, assign the usual strict inductive limit topology of the spaces $\mathcal{C}(K)$, where the latter is the space of all functions in $\mathcal{C}\left(\mathbf{R}_{+}\right)$ with support contained in the compact set $K$, with the topology defined by the supremum norm. Then, the topology of $\mathcal{C}_{\sigma \kappa}\left(\mathbf{R}_{+}, E\right)$ is defined by the fundamental system of neighborhoods of the form $\left\{f \mid\left(\forall e^{\prime} \in U^{\circ}\right)\left(t \mapsto\left\langle f(t), e^{\prime}\right\rangle \in V\right)\right\}$, as $U$ ranges over $\mathcal{U}(E)$ and $V$ over $\mathcal{U}\left(\mathcal{C}_{\kappa o}\left(\mathbf{R}_{+}\right)\right)$. Call a semigroup $(E, T)$ with response in $\mathcal{C}_{\sigma \kappa}\left(\mathbf{R}_{+}, E\right)$ a finite-response semigroup.

$\mathcal{C}_{\sigma \kappa}\left(\mathbf{R}_{+}, E\right)$ and $\mathcal{C}_{\kappa}\left(\mathbf{R}_{+}, E\right)$ do in fact agree, algebraically as well as topologically, when $E$ is a Banach space. In particular, they agree when $E=\mathbf{K}$, so that $\mathcal{C}_{\sigma \kappa}\left(\mathbf{R}_{+}\right)=\mathcal{C}_{\boldsymbol{K}}\left(\mathbf{R}_{+}\right)$. Thus, we cannot hope to get an $\mathbf{R}_{+}$-ring generator which admits precisely those responses in $\mathcal{C}_{\sigma \kappa}\left(\mathbf{R}_{+}, E\right)$. We note also that $C_{\sigma \kappa}^{\prime}\left(\mathbf{R}_{+}\right)$is the space of all Radon measures on $\mathbf{R}_{+}$[H7, Ch. 4, §4].

We may alternately take $m=\infty$ here and work with the space $\mathcal{C}_{\sigma \kappa}^{\infty}\left(\mathbf{R}_{+}, E\right)$ of all infinitely differentiable functions in $\mathcal{C}_{\sigma \kappa}\left(\mathbf{R}_{+}, E\right)$. The dual $\mathcal{C}_{\kappa}^{\prime \infty}\left(\mathbf{R}_{+}\right)$of $\mathcal{C}_{\kappa}^{\infty}\left(\mathbf{R}_{+}\right)$(often denoted $\mathcal{D}^{\prime}$ in the literature), has been used as the basis in some approaches to continuous time systems (e.g., [K3]).

3.23 Other Useful Examples One may develop a host of other examples. We mention just two. Starting with periodic functions and their associated distributions [B2], a theory of periodic semigroups may be developed. Similarly, we may develop a theory of semigroups with response rapidly decaying at infinity by using the space $\mathcal{S}\left(\mathbf{R}_{+}, E\right)$ of functions in $C\left(\mathbf{R}_{+}, E\right)$ such that $\lim _{t \rightarrow \infty}\left(1+t^{2}\right) f(t)=0$ for all natural numbers $k$. The seminorms defining the topology are of the form $f \mapsto \sup _{t \in \mathbf{R}_{+}} \alpha\left(\left(1+t^{2}\right)^{k} f(t)\right)$, with $\alpha$ ranging over continuous seminorms on $E$ and $k$ ranging over the natural numbers. See [H7, Chap. 2, Example 11], for details on the related spaces.

3.24 Simple Examples It is also possible to construct some extremely simple examples. For example, let $\operatorname{Con}\left(\mathbf{R}_{+}, \mathrm{E}\right)$ denote the space of all constant functions in $\mathcal{C}\left(\mathbf{R}_{+}, E\right)$, endowed with the norm $f \mapsto|f(0)|$. Clearly, $\operatorname{Con}\left(\mathbf{R}_{+}, \mathrm{E}\right) \cong \mathbf{K}$ under the association $f \mapsto f(0)$. Nonetheless, Con satisfies all of the criteria for an $\mathbf{R}_{+}$-ring generator. Note also that $\operatorname{Con}^{\prime}\left(\mathbf{R}_{+}\right) \cong \mathbf{K}$, and $\delta_{s}=\delta_{t}$ for any $s, t \in \mathbf{R}_{+}$. In other words, suitably interpreted, $\mathbf{K}$ itself is an $\mathbf{R}_{+}$-ring generator, albeit a trivial and rather uninteresting one.

\section{Behavior}

In order to discuss behavior properly, we need to fix two parameters. First of all, a base category $\mathcal{K}$ of l.c.s.'s from which all of the spaces of interest will be taken must be identified. $\mathcal{K}$ will always be taken to be a full subcategory of the category $\mathbf{L C S}$ of all locally convex spaces, with continuous linear maps as morphisms. Second, a base $\mathbf{R}_{+}$-ring $R$ must be established. Its underlying space is a $\mathcal{K}$-object, upon which all dynamics will be based. Having established these two parameters, the category $\operatorname{Dyn}(R, \mathcal{K})$ is defined to be that whose objects are the $(R)$-semigroups $(E, T)$ such that $E$ is a $\mathcal{K}$ object and whose morphisms are just the associated semigroup morphisms, which are termed dynamorphisms, in agreement with [AM1]. (Thus, $\operatorname{Dyn}(R, \mathcal{K})$ is just the category $\operatorname{CSG}(R, \mathcal{K})$ described earlier.) An $(R, \mathcal{K})$-system is a 6-tuple $(Q, T, I, Y, g, h)$ such that $(Q, T)$ is 
an $(R, \mathcal{K})$ semigroup, $I$ and $Y$ are objects of $\mathcal{K}$, and $g: I \rightarrow Q$ and $h: Q \rightarrow Y$ are continuous linear maps. It should be remembered that to ensure that $\mathbf{g}_{T}$ is densely defined for each $(R, \mathcal{K})$ system, we must either take $R$ to be sequentially complete or else require each $\mathcal{K}$ object to be sequentially complete. However, these requirements do not affect the abstract constructions, since the infinitesimal generator is never explicitly utilized.

4.1 Definitions - Free and Cofree Dynamics It is now necessary to make precise the extensions, to the continuous-time case, of free and cofree dynamics as presented for discrete time in Section 1 . Let $I$ be a $\mathcal{K}$ object. A free $(R, \mathcal{K})$-dynamics over $I$ is an $(R, \mathcal{K})$-dynamics $\left(I^{\$}, \Sigma^{\$}\right)$ together with a $\mathcal{K}$ morphism $\eta: I \rightarrow I^{\$}$ such that for any $(R, \mathcal{K})$-dynamics $(Q, T)$ and $\mathcal{K}$ morphism $g: I \rightarrow Q$, there is a unique dynamorphism $\rho:\left(I^{\$}, \Sigma^{\$}\right) \rightarrow(Q, T)$ such that the following diagram commutes.
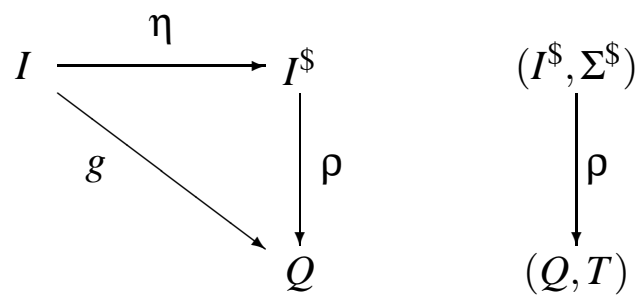

(The triangle on the left says that $g=\rho \circ \eta$ as $\mathcal{K}$ morphisms, while the arrow on the right says that $\rho$ is also a dynamorphism.)

Dually, let $Y$ be a $\mathcal{K}$ object. A cofree $(R, \mathcal{K})$-dynamics over $Y$ is an $(R, \mathcal{K})$-dynamics $\left(Y_{\$}, \Sigma_{\$}\right)$ together with a $\mathcal{K}$ morphism $\varepsilon: Y_{\$} \rightarrow Y$ such that for any other $(R, \mathcal{K})$-dynamics $(Q, T)$ and $\mathcal{K}$ morphism $h: Q \rightarrow Y$, there is a unique dynamorphism $\sigma:(Q, T) \rightarrow\left(Y_{\$}, \Sigma_{\$}\right)$ such that the following diagram commutes.
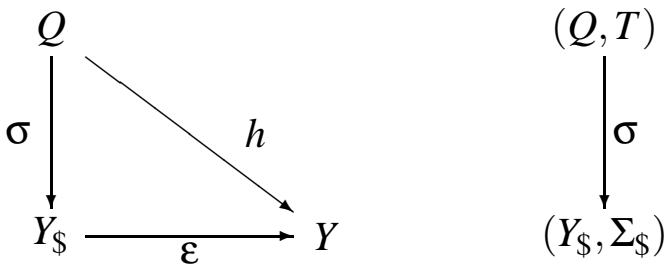

If they exist, free dynamics and cofree dynamics are unique up to isomorphism (see e.g., [HS, Prop. 26.7]), so we may speak of the free dynamics and the cofree dynamics, without essential ambiguity.

Let $M=(Q, T, I, g, Y, h)$ be an $(R, \mathcal{K})$-system. If both free dynamics over $I$ and cofree dynamics over $Y$ exist, then $\rho$ is called the reachability map, $\sigma$ the observability map, and $\sigma \circ \rho$ the behavior 
of $M$. Thus, to construct the behavior of $M$, it suffices to construct the free dynamics over $I$ and the cofree dynamics over $Y$, relative to $(R, \mathcal{K})$.

\section{Construction of Free Dynamics}

To construct free $(R, \mathcal{K})$-dynamics for a specific pair $(R, \mathcal{K})$, it is easiest to first perform the necessary constructions for $\mathcal{K}=\mathbf{L C S}$, and then to extend the results to partial completions of $\mathcal{K}$.

Let $R$ be an $\mathbf{R}_{+}$-ring, and let $I$ be any 1.c.s.. $R \otimes_{c} I$ denotes the tensor product of $R$ and $I$ (as vector spaces), equipped with the strongest locally convex topology making the canonical bilinear mapping $p: R \times I \rightarrow R \otimes I$ c-hypocontinuous. (We reserve the symbol $p$ to denote this mapping.) Recalling that $*_{R}$ denotes the ring multiplication of $R$, define the bilinear mapping $*_{R, I}: R \times\left(R \otimes_{c}\right.$ $I) \rightarrow R \otimes_{c} I$ by $(r, s \otimes i) \mapsto\left(r *_{R} s\right) \otimes i$. It is well-known that it is an $R$-module action [CE, Ch. 2]; we need to establish that it is an $(R)$-module action by demonstrating its c-hypocontinuity.

4.2 Lemma Let $R$ be an $\mathbf{R}_{+}$-ring and let $I$ be a l.c.s.. Then $R \otimes_{c} I$ is an $(R)$-module under the action $*_{R, I}$.

PROOF: In view of 3.3, it suffices to show that it is hypocontinuous with respect to the precompact subsets of $R$ and finite subsets of $R \otimes_{c} I$.

We first establish that $*_{R, I}$ is hypocontinuous with respect to the precompact subsets of $R$. Using the corresponding trilinear mapping $*_{R} \times I: R \times R \times I \rightarrow R \otimes_{c} I$ given by $R \times R \times I \stackrel{1 \times p}{\longrightarrow} R \times\left(R \otimes_{c}\right.$ $I) \stackrel{*_{R} \otimes I}{\longrightarrow} R \otimes_{c} I$, and given $V \in \mathcal{U}\left(R \otimes_{c} I\right)$, it must be established that (i) given any $K_{1}, K_{2} \in \mathcal{P}(R)$, there is a $U \in \mathcal{U}(I)$ such that $\left(*_{R} \times I\right)\left(K_{1} \times K_{2} \times U\right) \subseteq V$, and that (ii) given any $K_{1} \in \mathcal{P}(R)$ and $K_{3} \subseteq \mathcal{P}(I)$, there is a $W \in \mathcal{U}(R)$ such that $\left(*_{R} \times I\right)\left(K_{1} \times W \times K_{3}\right) \subseteq V$.

To show claim (i), let $V, K_{1}$, and $K_{2}$ be as stated. By 3.3(b), $K_{1} * K_{2}$ is precompact. Therefore, there is a $U \in \mathcal{U}(I)$ such that $p\left(\left(K_{1} * K_{2}\right) \times U\right) \subseteq V$. Since $p\left(\left(K_{1} * K_{2}\right) \times U\right)=\left(*_{R} \times I\right)\left(K_{1} \times K_{2} \times\right.$ $U)$, (i) is proved.

To show claim (ii), let $V, K_{1}$, and $K_{3}$, be as stated. Since $p$ is c-hypocontinuous, there is a $Y \in$ $\mathcal{U}(R)$ such that $p\left(Y \times K_{3}\right) \subseteq V$. Since multiplication in $R$ is c-hypocontinuous, there is a $U \in \mathcal{U}(R)$ such that $K_{1} * U \subseteq Y$, so $p\left(\left(K_{1} * U\right) \times K_{3}\right) \subseteq V$. As $p\left(\left(K_{1} * U\right) \times K_{3}\right)=\left(*_{R} \times I\right)\left(K_{1} \times U \times K_{3}\right)$, (ii) is proved.

The hypocontinuity of $*_{R, I}$ with respect to the finite subsets of $R \otimes_{c} I$ follows immediately from claim (ii) and the fact that $*_{R}$ is commutative.

We let $\Sigma^{R} \otimes I$ denote the semigroup action corresponding to $*_{R, I}$; i.e., $\mathbf{T}_{*_{R, I}}$. Clearly, $\left(\left(\Sigma^{R} \otimes\right.\right.$ $I)(t))(r \otimes i)=\left(\left(\delta_{t} * r\right) \otimes i\right)$. Now, given a 1.c.s. $I$, define $\eta: I \rightarrow R \otimes_{c} I$ by $i \mapsto \delta_{o} \otimes i$. $\eta$ is surely continuous, as it is the restriction of $p: R \times I \rightarrow R \otimes I$ to $R \times\left\{\delta_{o}\right\}$. We have the following fundamental result.

4.3 Theorem Let $R$ be an $\mathbf{R}_{+}$-ring, and let $I$ be a l.c.s.. Then $\left(\left(R \otimes_{c} I, \Sigma^{R} \otimes I\right), \eta\right)$ is a free $(R)$-semigroup over I (i.e., it is a free $(R, \mathbf{L C S})$-dynamics over $I)$. Given another $(R)$-semigroup 
$(Q, T)$ and continuous linear mapping $g: I \rightarrow Q$, the unique $(R)$-semigroup morphism $\rho$ making the diagram below commute is defined by $r \otimes i \mapsto \mathbf{b}_{T, R}(r, g(i))$.

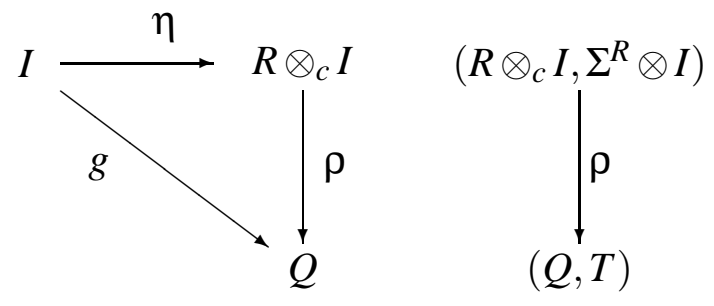

PROOF: The proof is in terms of the associated $(R)$-module actions. It is well-known that $R \otimes I$ is a free $R$-module, with $\eta$ as defined [CE, Ch. 2]. By the above lemma, we know that it is also an (R)-module. It is clear that $R \times I \stackrel{1 \times g}{\longrightarrow} R \times Q \stackrel{b}{\longrightarrow} Q$ is c-hypocontinuous, whence $\rho$ is continuous.

Let $M=(Q, T, I, g, Y, h)$ be an $(R, \mathbf{L C S})$-system. The above construction defines the space of inputs and the reachability map for $M$. Let us first consider inputs in $\Delta(R) \otimes_{c} I$. View the element $\delta_{t} \otimes I$ as an impulse input applied to the system at time $-t$ with weight $i$. (Note the time reversal.) The response $\rho\left(\delta_{t} \otimes i\right)$ is the resulting state at time 0 . Thus, $\Delta(R) \otimes I$ is regarded as an input space of finite linear combinations of $I$-valued impulses occurring at times $\leq 0$. The reachability map $\rho$ gives the response at time 0 to such a train of impulses. That is, $\rho\left(\sum_{k=1}^{n} \delta_{t_{k}} \otimes i_{k}\right)=\sum_{k=1}^{n} \mathbf{b}_{T, R}\left(\delta_{t_{k}}, g\left(i_{k}\right)\right)=\sum_{k=1}^{n} T\left(t_{k}\right) g\left(i_{k}\right)$. Note the direct analogy to the discretetime case, in which an input may be regarded to be of the form $\left.\sum_{k=1}^{n} \delta_{k} \otimes i_{k}\left(=\ldots, i_{k}, \ldots, i_{1}, i_{0}\right)\right)$ and the response to be $\sum_{k=1}^{n} T(k) g\left(i_{k}\right)=\sum_{k=1}^{n} f^{k} g\left(i_{k}\right)$, where $f=T(1)$ [AM1]. The only difference, other than the topological considerations required for differentiation, is that the set of times at which an input is allowed to occur is the nonpositive reals $\mathbf{R}_{-}$in the continuous-time case (recall the time reversal) and the nonpositive integers $\mathbf{N}_{-}$in the discrete-time case, and that $f$ assumes the role of a continuous rather than discrete generator. Thus, if we first examine the input signals and reachability map of a continuous-time linear system in terms of its skeleton input set $\Delta(R) \otimes I$, we see that it is not all that different from its discrete-time counterpart. What does make continuoustime linear systems richer than their discrete-time counterparts is the ability to complete $\Delta(R)$ and $\Delta(R) \otimes I$ to obtain a much more diversified set of inputs, tailored to the specific situation.

Let $r \otimes i \in R \otimes_{c} I$. If $r=\delta_{t}$ for some $t$, we already know that $\rho(r \otimes i)=T(t) g(i)$. If $r \in R$ more generally, we can approximate $r$ as closely as desired by a sum of the form $\sum_{j=1}^{n} a_{j} \cdot \delta_{t_{j}}$ with $a_{j} \in \mathbf{K}$, since $\Delta(R)$ is dense in $R . \sum_{j=1}^{n} a_{j} \cdot \delta_{t_{j}} \otimes i$ then approximates $r \otimes i$. Since $\rho$ is continuous, $\rho(r \otimes i)$ is approximated by $\sum_{j=1}^{n} a_{j} \cdot T\left(t_{j}\right) g(i)$.

Now suppose that $r$ is represented by a function $\varphi_{r}: \mathbf{R}_{+} \rightarrow \mathbf{K}$. Then, using the density of $\Delta(R)$ in $R$, there must be a net of sums $\sum_{j=1}^{n_{\alpha}} a_{j} \cdot T\left(t_{j}\right) g(i)$ which converges to the $Q$-valued integral $\int_{0}^{\infty} \varphi_{r}(t) T(t) g(i) d t$. Regarding $\varphi_{r}(t)$ as an input signal to the system $M$, the time scale is reversed, so if we view $\varphi: \mathbf{R}_{-} \rightarrow \mathbf{K}$, the state at time $t=0$ is $q(0)=\int_{-\infty}^{0} T(-t)\left(\varphi_{r}(t) \cdot g(i)\right) d t$, which is in 
agreement with the classical result for finite-dimensional systems [PA, 6-1, Thm. 2]. The extension to a sum of the form $\sum_{k=1}^{n} r_{k} \otimes i_{k}$ of such inputs is by simple linearity. For general inputs of the form $\mu \otimes i \in R \otimes_{c} I$, this above formula also works, since we may view $(t \mapsto T(t)) \in \mathbf{L}_{c}(R, E)$, by 3.9, and then view $\int_{0}^{\infty} \mu T(t) g(i) d t \in E$ as the weakly continuous linear functional on $E^{\prime}$ given by $e^{\prime} \mapsto \mu\left(t \mapsto\left\langle T(t) g(i), e^{\prime}\right\rangle\right)$

It is useful to have a more concrete representation of the space $R \otimes I$. In harmony with the notion of vector-valued distribution of Schwartz [S2], we call $\mathbf{L}_{c}\left(R^{\prime}, I\right)$ the space of all I-valued $(R)$ distributions. An element $f \in \mathbf{L}\left(R^{\prime}, I\right)$ is termed finite dimensional if $f\left(R^{\prime}\right)$ is a finite-dimensional subspace of $I$, and the subspace consisting of these mappings is denoted $\mathbf{F}\left(R^{\prime}, I\right)$. Now, for any test function $\varphi \in R^{\prime}$ and $\mu \in \mathbf{L}_{c}\left(R^{\prime}, I\right)$, we have a natural action $\langle\mu, \varphi\rangle=\varphi(\mu) \in I$ and a natural injection $\Phi: R \otimes I \rightarrow \mathbf{F}\left(R^{\prime}, I\right)$ via $\mu \otimes i \mapsto(\varphi \mapsto\langle\mu, \varphi\rangle \cdot i)$. Conversely, any $f \in \mathbf{F}\left(R^{\prime}, I\right)$ has a unique decomposition of the form $\sum_{j=1}^{n} \mu_{j} \cdot i_{j}$ with each $\mu_{j} \in R^{\prime \prime}$ and each $i_{j} \in I$, so every $\sum_{j=1}^{n} \mu_{j} \otimes i_{j}$ is a representation of $f$ in $R^{\prime \prime} \otimes I$. Thus, if $R$ is c-complete (so $R^{\prime \prime}=R$ ), then the space of inputs $R \otimes I$ may be identified with the space of all finite-dimensional $I$-valued $(R)$-distributions.

If $I=\mathbf{K}^{m}$ for some finite $m$, then every linear map into $I$ is finite dimensional, so $R \otimes_{c} I \cong R^{m}$, both algebraically and topologically. However, if $I$ is not finite dimensional, then $\mathbf{F}\left(R^{\prime}, I\right)$ is always a proper subset of $\mathbf{L}\left(R^{\prime}, I\right)$, and the topology induced on $\mathbf{F}\left(R^{\prime}, I\right)$ by $R \otimes_{c} I$ is generally strictly finer than the topology induced as a subspace of $\mathbf{L}_{c}\left(R^{\prime}, I\right)$. To understand this situation further, we pursue construction of a free $(R, \mathcal{K})$-dynamics in the case that $\mathcal{K}$ is $\widetilde{\mathbf{L C S}}$ for some partial completion operator $\sim$. In the abstract sense, all that need be done is to complete the spaces involved in the construction for the LCS case given in 4.3. However, there are a few details which must be addressed to ensure that this extension is well-defined. We proceed as follows.

4.4 Lemma Let $E$ and $F$ be l.c.s.'s such that for any linear $f: E \rightarrow F$, if $f_{\mid A}(f$ restricted to $A)$ is continuous for each $A \in \mathcal{P}(E)$, then $f \in \mathbf{L}(E, F)$. Then for any partial completion operator ${ }^{\sim}$, $\mathbf{L}_{c}(E, F)$ is ${ }^{\sim}$-complete whenever $F$ is.

Proof: For the case of ${ }^{\sim}=\widehat{ }$, this is just Theorem 32.2 of [T] specialized to precompact sets. However, the proof in [T] goes through for any partial completion operator with only inessential changes. We therefore omit the proof, and refer the reader to [T] instead.

4.5 Proposition Let $E$ be an almost polar reflexive l.c.s. such that $E^{\prime}$ is complete, and let $F$ be any l.c.s.. Then, for any partial completion operator ${ }^{\sim}, \mathbf{L}_{c}(E, F)$ is ${ }^{\sim}$-complete whenever $F$ is.

Proof: We show that $E$ satisfies the conditions of the above lemma. Let $f: E \rightarrow F$ be a linear map which is continuous on each $K \in \mathcal{P}(E)$. Then, in particular, $f$ sends precompact sets into precompact sets. Let $f^{*}: F^{*} \rightarrow E^{*}$ denote the algebraic transpose of $f$. Now each $g \in f^{*}\left(F^{\prime}\right)$ maps precompact subsets of $E$ into precompact subsets of $\mathbf{K}$, just by definition of $f^{*}$. Each such $g$ is continuous on precompact sets, since $\mathbf{K}$ is locally compact. Thus $g \in \widehat{E}^{\prime}$ by Grothendieck's completion theorem $[\mathrm{K} 6, \S 21,9,(2)]$. Since $E^{\prime}$ is already complete, we must in fact have a continuous $f^{\prime}: F^{\prime} \rightarrow E^{\prime}$, and so $f^{\prime \prime}: E^{\prime \prime} \rightarrow F^{\prime \prime}$ is also continuous. Since $E$ is almost polar reflexive, this may be written as $f_{\mid E}^{\prime \prime}: E \rightarrow F^{\prime \prime}$. Then, noting that $F^{\prime \prime}$ induces a topology on $F$ which is finer than the 
initial topology, we have that $f$ is continuous, as required.

4.6 Proposition Let $R$ be an $\mathbf{R}_{+}$-ring, and let $(E, b)$ be an $(R)$-module. Let $\sim$ be a partial completion operator. Then b extends uniquely to an $(R)$-module action $R \times \widetilde{E} \rightarrow \widetilde{E}$.

PROOF: First extend the codomain of $b$ to be $\widetilde{E} ; b: R \times E \rightarrow \widetilde{E}$. Next, convert this to a linear mapping $\bar{b}: E \rightarrow \mathbf{L}_{c}(R, \widetilde{E})$. By the above theorem, $\left(\mathbf{L}_{c}(R, \widetilde{E})\right)^{\sim}=\mathbf{L}_{c}(R, \widetilde{E})$, so $\bar{b}$ extends to $\widetilde{E} \rightarrow$ $\mathbf{L}_{c}(R, \widetilde{E})$. An application of 3.3(a) now shows that the corresponding bilinear mapping $R \times \widetilde{E} \rightarrow \widetilde{E}$ is c-hypocontinuous.

Let $\sim$ be a partial completion operator, let $I$ be a l.c.s., and let $R$ be a ${ }^{\sim}$-complete $\mathbf{R}_{+}$-ring. Denote by $\widetilde{*}_{R, I}$ the unique extension of the $(R)$-module action $*_{R, I}$ to $R \widetilde{\otimes}_{c} I$ guaranteed by the above theorem, and let $\Sigma^{R} \widetilde{\otimes}_{c} I$ denote the associated $(R)$-semigroup action. $\eta: I \rightarrow R \widetilde{\otimes}_{c} I$ denotes the extension of the map $\eta$ defined previously. We then have the following extension of 4.4.

4.7 Theorem Let $\sim$ be a partial completion operator, let I be $a^{\sim}{ }^{\sim}$-complete l.c.s., and let $R$ be an $\mathbf{R}_{+}$-ring. Then $\left(\left(R \widetilde{\otimes}_{c} I, \Sigma^{R} \widetilde{\otimes}_{c} I\right), \sim \eta\right)$ is a free $(R)$-semigroup over I (i.e., it is a free $(R, \widetilde{\mathbf{L C S}})$ dynamics over $I)$. For $(Q, T)$ an $\left(R,{ }^{\sim} \mathbf{L C S}\right)$-dynamics and $g: I \rightarrow Q$ a continuous linear mapping, the unique morphism required is just $\widetilde{\rho}$ as shown below, with $\rho$ defined as in 4.4 .

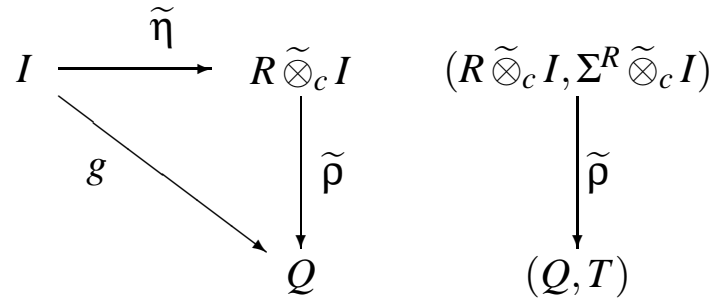

PROOF: Follows immediately from 4.4 and the previous proposition.

Unfortunately, we do not have a completely satisfactory concrete representation of $R \widetilde{\otimes}_{c} I$ is the most general case. The best that we can do is to study its relationship to the projective tensor product, about which a great deal more is known. Specifically, the projective tensor product $R \otimes_{\pi} I$ carries the strongest locally convex topology making the canonical bilinear mapping $p: R \times I \rightarrow$ $R \otimes I$ continuous [K7, §41]. In general, this topology is strictly weaker than the topology on $R \otimes_{c} I$. Nontheless, there are substantial ties between these two tensor products, as shown by the following result.

4.8 Proposition Let $E$ and $F$ be two arbitrary l.c.s.'s.

(a) The precompact subsets of $E \otimes_{\pi} F$ are precisely the subsets of closed absolutely convex hulls of sets of the form $K_{1} \otimes K_{2}$, with $K_{1}$ and $K_{2}$ precompact. 
(b) The precompact subsets of $E \otimes_{c} F$ and $E \otimes_{\pi} F$ are identical.

(c) The continuous bijection $E \otimes_{c} F \rightarrow E \otimes_{\pi} F$ may be extended to a continuous bijection $E \check{\otimes}_{c} F \rightarrow E \check{\otimes}_{\pi} F$, whose restriction to each precompact subset is a topological isomorphism.

(d) If $E$ is polar reflexive, then the canonical injection $\Phi: E \otimes_{\pi} F \rightarrow \mathbf{L}_{c}\left(E^{\prime}, F\right)$ is a continuous embedding whose image is $\mathbf{F}\left(E^{\prime}, F\right)$.

(e) If $E$ and $F$ are $(P F)$ spaces, then $E \otimes_{c} F$ and $E \otimes_{\pi} F$ carry the same topology.

PROOF: (a) Since $E \otimes_{\pi} F$ carries the strongest locally convex topology making the natural projection $p: E \times F \rightarrow E \otimes F$ continuous, the precompact subsets of $E \otimes_{\pi} F$ must be exactly those sets $K$ whose inverse image $p^{-1}(K)$ is precompact in $E \times F$. Since the precompact subsets of $E \times F$ are precisely the products of precompact sets in $E$ and $F$, the result follows.

(b) Since $p: E \times F \rightarrow E \otimes_{c} F$ is c-hypocontinuous, we have immediately that the closed absolutely convex hull of $p\left(K_{1} \times K_{2}\right)$ is precompact in $E \otimes_{c} F$. However, $E \otimes_{c} F$ carries a finer topology than does $E \otimes_{\pi} F$, and hence must have no more precompact sets. The result thus follows from (a).

(c) Follows immediately from (b), since a continuous bijection of compact spaces is necessarily a homeomorphism.

(d) Let $\beta: E \times F \rightarrow \mathbf{L}_{c}\left(E^{\prime}, F\right)$ given on elements by $(\mu, i) \mapsto(\varphi \mapsto\langle\mu, \varphi\rangle \cdot i)$ be the bilinear mapping corresponding to $\Phi$. For $K \in \mathcal{P}\left(E^{\prime}\right), V \in \mathcal{U}(F)$, we have $\beta\left(K^{\circ} \times V\right) \subseteq\{f \mid f(K) \subseteq V\}$, so that $\beta$ is continuous, and so, a fortiori, c-hypocontinuous. Thus $\Phi$ is continuous. In fact, if we let $F_{1} \in \mathbf{L}\left(E^{\prime}, F\right)$ denote the subspace consisting of the maps with a one-dimensional image (represented in $E \otimes_{c} F$ by elements of the form $\mu \otimes i$ ), then it is easy to see that $\beta$ is onto $F_{1}$, and that $\beta\left(K^{\circ} \times V\right)=\left\{f \in F_{1} \mid f(K) \subseteq V\right\}$, so that $\beta$ is an open mapping. Translating back, this means precisely that $\Phi$ is an embedding. Since $E$ is polar reflexive, $E \otimes F=E^{\prime \prime} \otimes F=\mathbf{F}\left(E^{\prime}, F\right)$ by the standard characterization $[K 7, \S 43,1]$, whence $\Phi$ is surjective.

(e) If $E$ and $F$ are (PF) spaces, then every c-hypocontinuous bilinear mapping on $E \times F$ is continuous. The proof follows almost verbatim the standard one that every hypcontinuous bilinear mapping on (DF) spaces is continuous [K7, §40,2(10)]. It suffices to note that the compact subsets of (PF) spaces are correspond exactly to the bounded subspaces in their (DF) counterparts, and to substitute "compact" for "bounded" throughout.

From the preceding proposition, we may deduce that $R \widetilde{\otimes}_{c} I$ is naturally isomorphic to a subspace of $I$-valued $(R)$-distributions, provided that ${ }^{\sim} \leq^{\vee}$. The compact sets will be exactly those induced by $\mathbf{L}_{c}\left(\mathbf{R}_{+}, I\right)$. However, in the general case, the induced topology will be strictly finer, and any attempt to go beyond c-completion may run into injectivity problems. More to the point, it is certainly possible to extend the continuous injection $R \otimes_{c} I \rightarrow R \otimes_{\pi} I$ to the completions $R \widehat{\otimes}_{c} I \rightarrow$ $R \widehat{\otimes}_{\pi} I$, but there is no guarantee that this extension will be injective. If it were not injective, this would imply the existence of nonzero elements of $R \widehat{\otimes}_{c} I$ which correspond to the zero mapping in $\mathbf{L}\left(R^{\prime}, I\right)$, rendering any model of $R \widehat{\otimes}_{c} I$ as a subspace of $\mathbf{L}\left(R^{\prime}, I\right)$ somewhat meaningless. We know of no work addressing this issue, and we can offer no futher insight into it at the present time. 
Another characterization of $R \check{\otimes}_{c} I$ is provided in [H5]. There we show that if $I$ is polar reflexive, so too is $R \check{\otimes}_{c} I$, and the latter is naturally isomorphic to the polar dual of $\mathbf{L}_{c}\left(R, I^{\prime}\right)$. But in that framework as well, it seems impossible to obtain anything a concrete representation beyond ccompletion.

To obtain a truly concrete representation of $R \widehat{\otimes}_{c} I$, we can offer no better alternative than to restrict attention to those cases in which $R \otimes_{c} I=R \otimes_{\pi} I$. Item (e) of the preceding proposition provides such conditions; namely, require both $R$ and $I$ to be (PF) spaces. This is not as extreme a constraint as might first appear. We know from the previous section that most of the important examples of $\mathbf{R}_{+}$-rings are (PF) spaces. Furthermore, whenever $I$ is a Banach space or the dual of a Fréchet space, it will be a (PF) space as well.

Since the natural injection $R \otimes_{\pi} I \rightarrow \mathbf{L}_{c}\left(R^{\prime}, I\right)$ is an embedding, and since $\mathbf{L}_{c}\left(R^{\prime}, I\right)$ is complete whenever $I$ is, we know that $R \widetilde{\otimes}_{c} I$ will be a (topological) subspace of $\mathbf{L}_{c}\left(R^{\prime}, I\right)$, the space of all $I$-valued $(R)$-distributions. It is pertinent to ask when it will be all of $\mathbf{L}_{c}\left(R^{\prime}, I\right)$. It

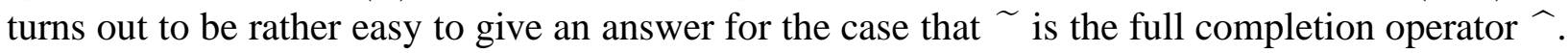
Following [K7, §43,1], we say that an 1.c.s. $E$ has the approximation property if $\mathbf{F}(E, E)$ is dense in $\mathbf{L}_{c}(E)$.

4.9 Proposition Let $R$ be a complete $\mathbf{R}_{+}$-ring with the approximation property, and let I be a complete lcs.

(a) $R \widehat{\otimes}_{\pi} I$ is naturally isomorphic (topologically as well as algebraically) to $\mathbf{L}_{c}\left(R^{\prime}, I\right)$.

(b) If $R$ is of the form $\mathcal{F}^{\prime}\left(\mathbf{R}_{+}\right)$, with $\mathcal{F}\left(\mathbf{R}_{+}\right)$an $\widetilde{\mathbf{H}}^{m}$ space in the sense of Schwartz [S1], then $R$ has the approximation property.

PROOF: (a) This follows immediately from [K7, §43,1(1)] and 4.5.

(b) For a proof that every $\widetilde{\mathbf{H}}^{m}$ space has the approximation property, see [S1, Prop. 16]. However, if $R^{\prime}$ has the approximation property, then so too does $R$. This follows from [K7, §43,4(9)], noting that in that statement and its proof, "quasi-complete" may be everywhere replaced with "c-complete".

We note that all of the $\mathbf{R}_{+}$-ring examples given in the previous section have the approximation property, although we do not have any general approach for establishing those results beyond (b) above. For related work on the approximation property and spaces of distributions, consult [S2].

\section{Cofree $(R)$-Modules and Dynamics}

The cofree constructions are much simpler than their free counterparts. We proceed as follows. Let $R$ be an $\mathbf{R}_{+}$-ring, and let $Y$ be a 1.c.s.. Define the bilinear mapping $\mathbf{Z}(R, Y): R \times \mathbf{L}_{c}(R, Y) \rightarrow$ $\mathbf{L}_{c}(R, Y)$ by $(r, f) \mapsto f(r *-)$. It is easy to see that $\mathbf{Z}(R, Y)$ defines an $R$-module action. We need to establish its c-hypocontinuity to show that it is in fact an $(R)$-module action. 
4.10 Lemma For any $\mathbf{R}_{+}$-ring $R$ and l.c.s. $Y, \mathbf{Z}(R, Y)$ is an $(R)$-module action on $\mathbf{L}_{c}(R, Y)$.

Proof: Let $W \in \mathcal{U}\left(\mathbf{L}_{c}(R, Y)\right)$; it suffices to take $W=\{f \mid f(K) \subseteq V\}$ for some $K \in \mathcal{P}(R)$ and $V \in$ $\mathcal{U}(Y)$. Let $g \in \mathbf{L}_{c}(R, Y)$. Since $g$ is continuous and multiplication in $R$ is c-hypocontinuous, there is a $U \in \mathcal{U}(R)$ such that $g(U * K) \subseteq V$. Thus $\mathbf{Z}(R, Y)(U \times\{g\}) \subseteq W$. Next, keep $V$ as above and let $K_{1} \in \mathcal{P}(R)$. Put $U=\left\{f \mid f\left(K * K_{1}\right) \subseteq V\right\}$. By 3.3(b), $K * K_{1}$ is precompact, so $U \in \mathcal{U}\left(\mathbf{L}_{c}(R, Y)\right)$. Note that $\mathbf{Z}(R, Y)\left(K_{1} \times U\right) \subseteq W$. Hence $\mathbf{Z}(R, Y)$ is hypocontinuous with respect to the precompact subsets of $R$ and the finite subsets of $\mathbf{L}_{c}(R, Y)$, so, by 3.3(a), it is c-hypocontinuous.

Let $R$ be an $\mathbf{R}_{+}$-ring and let $Y$ be a l.c.s.. We let $\left[\Sigma_{R}, Y\right]$ denote the semigroup action corresponding to $\mathbf{Z}(R, Y)$; i.e., $\mathbf{T}_{\mathbf{Z}(R, Y)}$. Clearly, $\left[\Sigma_{R}, Y\right](t) f=f\left(\boldsymbol{\delta}_{t} *-\right)$. Furthermore, if $R=\mathcal{F}^{\prime}\left(\mathbf{R}_{+}\right)$, then $\mathbf{L}_{c}(R, Y) \cong \mathcal{F}\left(\mathbf{R}_{+}, Y\right)$, and $\left[\Sigma_{R}, Y\right](t)$ corresponds to the left translation operator $\tau_{t}$. Define $\varepsilon: \mathbf{L}_{c}(R, Y) \rightarrow Y$ by $f \mapsto f\left(\boldsymbol{\delta}_{0}\right)$. Since $\varepsilon(f)=\mathbf{Z}(R, Y)\left(\boldsymbol{\delta}_{o}, f\right)(0)$, it is surely continuous.

4.11 Theorem Let $R$ be an $\mathbf{R}_{+}$-ring, let $\mathcal{K}$ be $\widetilde{\mathbf{L C S}}$ for some partial completion operator $\sim$ (including the identity completion operator), and let $Y$ be a $\mathcal{K}$ object. Then $\left(\left(\mathbf{L}_{c}(R, Y),\left[\Sigma_{R}, Y\right]\right), \varepsilon\right)$ is a cofree $(R)$-semigroup over $Y$ in $\mathcal{K}$. If $(Q, T)$ is any $(R)$-semigroup in $\mathcal{K}$ and $h: Q \rightarrow Y$ is a continuous linear mapping, then the unique $(R)$-semigroup morphism $\varepsilon:(Q, T) \rightarrow\left(\mathbf{L}_{c}(R, Y),\left[\Sigma_{R}, Y\right]\right)$ such that the following diagram commutes is given by $q \mapsto(r \mapsto h(b(r, q)))$.
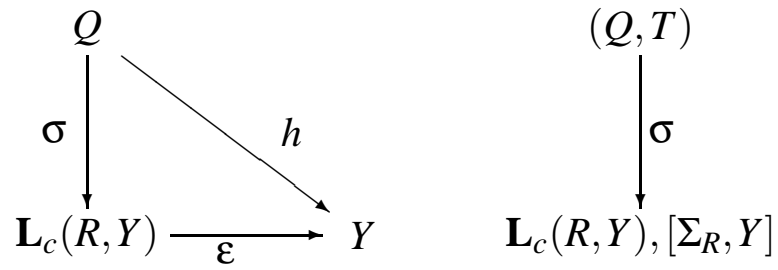

ProOF: It is well-known that $\mathbf{Z}(R, Y)$ is a cofree $R$-module over $Y$, with $\varepsilon$ as defined [CE, Ch. 2]. By the previous lemma, $\mathbf{Z}(R, Y)$ is an $(R)$-module. The continuity of $\sigma$ follows form the fact that $\sigma=Q \stackrel{\sigma_{Q}}{\longrightarrow} \mathbf{L}_{c}(R, Q) \stackrel{-. h}{\longrightarrow} \mathbf{L}_{c}(R, Y)$, where $\mathrm{v}_{Q}$ is the $\nu$ of 3.9. Since $\mathbf{L}_{c}(R, Y)$ is a $\mathcal{K}$ object whenever $Y$ is (4.8), upon translating to $(R)$-semigroups, the result is proved.

Let $M=(Q, f, I, g, Y, h)$ be an $(R, \mathcal{K})$-system. $\sigma$ of the above corollary is the observability map of $M$. Its interpretation is very simple. $\sigma(q)$ gives the output trajectory of the system for $t \geq 0$ with $q$ the state at $t=0$, and input 0 for all $t>0$. The output at $t \geq 0$ is just $\sigma(q)(t)$. Again, we have a close correspondence with the discrete-time case, where the space of output signals may be considered to be $Y_{\S}=\{\varphi \mid \varphi: \mathbf{N} \rightarrow Y\}$ as shown in the introduction, wheras in the continuous-time case it is a subset of $\left\{\varphi \mid \varphi: \mathbf{R}_{+} \rightarrow Y\right.$ continuously $\}$. 


\section{Complementary Directions}

It is possible to develop a realization theory for $(R, \mathcal{K})$-systems as well. As has been known for some time, defining the system $M$ to be reachable if its reachability map $\rho$ is surjective, and observable if its observability map $\sigma$ is injective, is not adequate in the continuous-time case, or even in the topologized discrete-time case [H1]. Rather, a satisfactory realization theory must be based upon more refined factorizations which take topology into account, such as (open surjections, injections), (surjections, embeddings), or (dense maps, closed embeddings). The appropriate algebraic formulation is that of an image-factorization system $(\mathbf{E}, \mathbf{M})$ in the category $\mathcal{K}$ [AM1]. The details of this formulation are not significantly different in principle from those presented in [H2], and so we do not pursue a complete formulation here, but rather we direct the reader to that reference. A detailed development may also be found in the technical report [H3].

It is possible to achieve a duality theory for continuous-time systems of the form developed in this work as well. However, the algebraic machinery required is the much more substantial enriched category theory [K4]. Basically, we are required to consider "structured" categories in which the set of morphisms forms not just a set, but a locally convex space. The details will be laid out in a separate report [H5].

\section{References}

[AM1] Arbib, M. A. and Manes, E. G., Foundations of system theory: Decomposable systems, Automatica - J. IFAC, 10(1974), 285-302.

[AM2] Arbib, M. A. and Manes, E. G., Adjoint machines, state-behavior machines, and duality, J. Pure Appl. Algebra, 6(1975), 313-344.

[AM3] Arbib, M. A. and Manes, E. G., Arrows, Structures, and Functors: The Categorical Imperative, Academic Press, New York, 1975.

[AM4] Arbib, M. A. and Manes, E. G., Intertwined recursion, tree transformations, and linear systems, Information and Control, 40(1979), 144-180.

[AM5] Arbib, M. A. and Manes, E. G., Machines in a category, J. Pure Appl. Algebra, 19(1980), 9-20.

[B1] Bainbridge, E. S., A unified minimal realization theory with duality, Technical Report 140, Logic of Computers Group, Department of Computer and Communication Sciences. University of Michigan, 1972, Also Ph.D. dissertation.

[B2] Beals, R., Advanced Mathematical Analysis, Springer-Verlag, New York, 1973.

[BDM] Bensoussan, A., Delfour, M. C., and Mitter, S. K., Representation and qualitative properties of infinite-dimensional linear systems, part I, Technical Report ESL-P-602, Elec- 
tronic Systems Laboratory, Department of Electrical Engineering, Massachusetts Institute of Technology, Cambridge, 1975.

[B3] Bourbaki, N., Eléments de Mathématique, Intégration, Hermann, Paris, 1965.

[B4] Bourbaki, N., Elements of Mathematics, General Topology, Addison-Wesley, Reading, MA, 1966.

[BSH] Brown, R. G., Sharpe, R. A., and Hughes, W. L., Lines, Waves, and Antennas, Ronald Press, New York, 1961.

[CE] Cartan, H. and Eilenberg, S., Homological Algebra, Princeton University Press, Princeton, 1956.

[DS] Dunford, N. and Schwartz, J. T., Linear Operators, Part 1: General Theory, Wiley Interscience, New York, 1958.

[EKKK] Ehrig, H., Kiermeier, K. D., Kreowski, H.-J., and Kühnel, W., Universal Theory of Automata, Teubner, Berlin, 1974.

[EK] Ehrig, H. and Kreowski, H.-J., Systematic approach to reduction and minimization in automata and system theory, J. Comput. System Science, 12(1976), 269-303.

[G] Goguen, J. A., Discrete-time machines in closed monoidal categories. I, J. Comput. System Science, 10(1975), 1-43.

[H1] Hegner, S. J., Duality theory for discrete-time linear systems, J. Comput. System Sci., 17(1978), 116-143.

[H2] Hegner, S. J., Linear decomposable systems in continuous time, SIAM J. Math. Anal., 12(1981), 243-273.

[H3] Hegner, S. J., Algebraic representation of classes of $\left(C_{o}\right)$ semigroups with applications to system theory, 1987, unpublished preliminary manuscript.

[H4] Hegner, S. J., Algebraic representation of classes of $\left(C_{o}\right)$ semigroups and the behavior of inhomogeneous linear differential equations, 1989, manuscript in preparation.

[H5] Hegner, S. J., An enriched category approach to continuous-time linear systems with duality, 1989, manuscript in preparation.

[H6] Helton, J. W., Systems with infinite-dimensional state space: The Hilbert space approach, Proc. IEEE, 64(1976), 145-160.

[HS] Herrlich, H. and Strecker, G. E., Category Theory, Allyn and Bacon, Boston, 1973. 
[H7] Horváth, J., Topological Vector Spaces and Distributions, Volume I, Addison-Wesley, Reading, MA, 1966.

[K1] Kalman, R. E., Advanced theory of linear systems, in: Topics in Mathematical System Theory (Kalman, R. E., Falb, P. L., and Arbib, M. A., eds.), McGraw-Hill, New York, 1969.

[KH] Kalman, R. E. and Hautus, M. L. J., Realization of continuous-time linear dynamical systems: Rigorous theory in the style of Schwartz, in: Ordinary Differential Equations, 1971 NRL-MRC Conference (Weiss, L., ed.), pp. 151-164, Academic Press, New York, 1972.

[K2] Kamen, E. W., A distributional-module theoretic representation of linear dynamical continuous-time systems, Technical Report 6650-24, Information Systems Laboratory, Stanford Electronic Laboratories, 1971, Also Ph.D. dissertation.

[K3] Kamen, E. W., Module structure of infinite-dimensional systems with applications to controllablity, SIAM J. Control Optimization, 14(1976), 389-408.

[K4] Kelly, G. M., Basic Concepts of Enriched Category Theory, Cambridge University Press, Cambridge, England, 1982.

[K5] Kōmura, T., Semigroups of operators in locally convex spaces, J. Functional Analysis, 2(1968), 248-296.

[K6] Köthe, G., Topological Vector Spaces I, Springer-Verlag, New York, 1969.

[K7] Köthe, G., Topological Vector Spaces II, Springer-Verlag, New York, 1979.

[PA] Padulo, L. and Arbib, M. A., System Theory: A Unified Approach to Continuous and Discrete Systems, Hemisphere, Washington, 1974.

[S1] Schwartz, L., Espaces de fonctions différentiables à valeurs vectorielles, J. Analyse. Math., 4(1954-55), 88-148.

[S2] Schwartz, L., Théorie des distributions à valeurs vectorielles, Chap. 1, Ann. Inst. Fourier (Grenoble), 7(1957), 1-141.

[S3] Schwartz, L., Théorie des Distributions, troisième édition, Hermann, Paris, 1966.

[T] Treves, F., Topological Vector Spaces, Distributions, and Kernels, Academic Press, New York, 1967.

[W] Waelbroeck, L., Les semi-groupes différentiables, in: Deuxième Colloque sur l'Analyse Fonctionelle, pp. 97-103, Centre Belge de Recherche Mathématique, 1964.

[Y] Yamamoto, Y., Module structure of constant linear systems and its application to controllablity, J. Math. Anal. Appl., 83(1981), 411-437. 


\section{Appendix - Polar Reflexivity and Ascoli's Theorem}

With the aid of Ascoli's theorem, we obtain some very useful results relating precompactness and equicontinuity of $\mathbf{L}_{c}(E, F)$ when $E$ is almost polar reflexive.

A.1 Theorem - Characterization of Precompactness in $\mathbf{L}_{c}(E, F)$ Let $E$ and $F$ be l.c.s.'s, and suppose that $E$ is almost polar reflexive. Then $H \subseteq \mathbf{L}_{c}(E, F)$ is precompact if and only if the following two conditions are satisfied.

(a) $H$ is equicontinuous.

(b) $H(K)$ is precompact for each $K \in \mathcal{P}(E)$.

Proof: ((a), (b) $\Rightarrow H$ precompact) is a special case of Ascoli's Theorem [B4, Ch. X, §2.5, Thm. 2].

( $H$ precompact $\Rightarrow($ b)) Let $K \in \mathcal{P}(E)$ and let $U \in \mathcal{U}(F)$. Since $H$ is precompact, there are $h_{1}, \ldots, h_{n} \in H$ such that given any $h \in H, h-h_{i} \in\{f \mid f(K) \subseteq U / 2\}$ for some $i \in[1, n]$. Trivially $\left\{h_{1}, \ldots, h_{n}\right\}$ is equicontinuous, so there is a $V \in \mathcal{U}(E)$ such that $h_{i}(V) \subseteq V / 2$ for all $i \in[1, n]$. Since $K$ is precompact, there are $k_{1}, \ldots, k_{m} \in K$ such that given any $k \in K, k-k_{i} \in V$ for some $i \in[1, m]$. Now let $x \in H(K)$; then $x=h(k)$ for some $h \in H$ and $k \in K$. By the preceding there are $h_{i}$ and $k_{j}$ such that $h-h_{i} \in\{f \mid f(K) \subseteq U / 2\}$ and $k-k_{j} \in V$. But then $h(k)-h_{i}\left(k_{j}\right)=$ $\left(h(k)-h_{i}(k)\right)+\left(h_{i}(k)-h_{i}\left(k_{j}\right)\right) \in U / 2+U / 2 \subseteq U$. Hence $\left\{h_{i}\left(k_{j}\right) \mid i \in[1, n] \wedge j \in[1, m]\right\} \subseteq H(K)$ is a finite set of points which are $U$-close to any $x \in H(K)$. Thus $H(K)$ is precompact.

( $H$ precompact $\Rightarrow$ (a)) Let $H \subseteq \mathbf{L}_{c}(E, F)$ be precompact; we must show that it is equicontinuous. First note that there is a natural embedding ' $: \mathbf{L}_{c}(E, F) \rightarrow \mathbf{L}_{\varepsilon}\left(F^{\prime}, E^{\prime}\right)$, where $\varepsilon$ defines the equicontinuous subsets of $F^{\prime}$. Since ' is continuous, $H^{\prime} \subseteq \mathbf{L}_{\varepsilon}\left(F^{\prime}, E^{\prime}\right)$ is also precompact, and since $\varepsilon$ defines precompact sets $[\mathrm{K} 6, \S 21,6(3)]$, Ascoli's Theorem may be invoked to deduce that $H^{\prime}(x)$ is precompact in $E^{\prime}$ for each $x \in F^{\prime}$. Also, by ( $H$ precompact $\left.\Rightarrow(b)\right)$ above, $H(K)$ is precompact for each $K \in \mathcal{P}(E)$, so $H^{\prime}$ is an equicontinuous subset of $\mathbf{L}\left(F^{\prime}, E^{\prime}\right)$. Hence we may apply Ascoli's Theorem again to deduce that $H^{\prime}$ is precompact in $\mathbf{L}_{c}\left(F^{\prime}, E^{\prime}\right)$. Now use ( $H$ precompact $\left.\Rightarrow(b)\right)$ once again to ascertain that $H^{\prime}(K)$ is precompact for each $K \in \mathcal{P}\left(F^{\prime}\right)$. Finally, transpose again to get that $H^{\prime \prime}$ is equicontinuous in $\mathbf{L}\left(E^{\prime \prime}, F^{\prime \prime}\right)$. Since $E$ is almost polar reflexive, we have that $E \rightarrow E^{\prime \prime}$ is continuous, so $H_{\mid E}^{\prime \prime} \subseteq \mathbf{L}\left(E, F^{\prime \prime}\right)$ is also equicontinuous. Since each $h \in H^{\prime \prime}$ is the bitranspose of an element of $H, H^{\prime \prime}(E) \subseteq F$. The topology which $F^{\prime \prime}$ induces on $F$ is finer than the initial topology, so $H_{\mid E}^{\prime \prime}=H \subseteq \mathbf{L}(E, F)$ is also equicontinuous.

A.2 Corollary - Characterization of c-hypocontinuity Let E, F, G be l.c.s.'s, and assume further that $E$ is almost polar reflexive. Let $b: E \times F \rightarrow G$ be a bilinear mapping which is hypocontinuous with respect to the precompact subsets of $E$ and the finite subsets of $F$. Then:

(a) b is c-hypocontinuous.

(b) $b\left(K_{1} \times K_{2}\right)$ is precompact for $K_{1} \in \mathcal{P}(E), K_{2} \in \mathcal{P}(F)$. 
(c) The transpose $b_{1}: E \times G^{\prime} \rightarrow F^{\prime}$ (resp. $b_{2}: G^{\prime} \times F \rightarrow E^{\prime}$ is hypocontinuous with respect to the precompact subsets of $E$ (resp. $F$ ).

Proof: (a) In view of the hypotheses, we may define a continuous linear mapping $\bar{b}: F \rightarrow$ $\mathbf{L}_{c}(E, G)$ defined on elements by $x \mapsto b(-, x)$. For any $K \in \mathcal{P}(F), \bar{b}(K)$ is precompact, and so equicontinuous by the above theorem. But the equicontinuity of $\bar{b}(K)$ is equivalent to the hypocontinuity of $b$ with respect to the precompact subsets of $F$, as was to be proved.

(b) Argue as above, noting that by the above theorem, $\bar{b}(K)$ maps precompact sets into precompact sets.

(c) This is just the transpose of (b). 